\title{
A dynamic framework for updating approximations with increasing or decreasing objects in multi- granulation rough sets
}

Hong Wang ( $\square$ whdw218@163.com )

Shanxi Normal University https://orcid.org/0000-0002-3300-1638

\section{Tao Guan}

Shanxi Normal University

\section{Research Article}

Keywords: Multi-granulation rough sets, Knowledge discovery, Incremental updating, Approximations

Posted Date: August 24th, 2021

DOl: https://doi.org/10.21203/rs.3.rs-531454/v1

License: (c) (1) This work is licensed under a Creative Commons Attribution 4.0 International License.

Read Full License 


\title{
A dynamic framework for updating approximations with increasing or decreasing objects in multi-granulation rough sets
}

\author{
Hong Wang*, Jingtao Guan \\ School of Mathematics and Computer Science, Shanxi Normal University, Shanxi, Linfen, 041000, P.R. China
}

\begin{abstract}
The data we need to deal with is getting bigger and bigger in recent years, and the same happens to multigranulation rough set, so updated schemes have been proposed with the variation of attributes or attribute values in multi-granulation rough sets, this paper puts forward a dynamic mechanism to update the approximations of multigranulation rough sets when adding or deleting objects. Firstly, the relationships between the original approximations and updated approximations are explored when adding or deleting objects in multi-granulation rough sets, and the dynamic processes of updating optimistic and pessimistic multi-granulation rough approximations are proposed. Secondly, two corresponding dynamic algorithms are proposed to update the lower and upper approximations of optimistic and pessimistic multi-granulation rough sets. Finally, a great quantity of experiments had been implemented, and the results indicate that two dynamic algorithms proposed are more effective than the static algorithm.
\end{abstract}

Keywords: Multi-granulation rough sets, Knowledge discovery, Incremental updating, Approximations

\section{Introduction}

As a data analysing and processing theory, rough set theory, set up by Polish scientist Z.Pawlak in 1982 [1], had made great progress in both theory and application practice as the scientific research of intelligent computing. Rough set theory is an effective tool to deal with imprecise, inconsistent and incomplete information without any prior knowledge, and it had been used in data mining [2], knowledge discovery [3], machine learning [4-6] and so on. As the basic computation of rough set, calculating the lower approximation and the upper approximation is an essential step for knowledge discovery and attribute reduction.

With the development of big data, the data we need to deal with in recent years is updating constantly, however, the original method can not recognise updated knowledge in time, which causes a problem: How do we get efficient results from fast data updating? Many scholars have done a lot of research about it, and they found that the updated data are linked to the original data. Thereby we can obtain updated knowledge via the relationship between them

\footnotetext{
${ }^{*}$ Corresponding author

Email addresses: whdw218@163.com (Hong Wang), 1121208261@qq.com (Jingtao Guan)
} 
and existing knowledge, a lot of time and space will be saved. There are three main situations as follows and we can obtain updated knowledge via set operation or matrix operation.

Updating data with the variation of objects. Shu et al. proposed the incremental method of dynamic feature selection by updating the dependency function [7]. An attribute reduction and incremental algorithm of decision rule based on the decision matrix were proposed by Fan et al. [8]. Zhang et al. came up with a dynamic neighborhood rough set model to deal with the dynamic change of numerical information system [9]. Chen put forward an incremental algorithm for data when objects are added or deleted [10] based on the variable precision rough set model. An incremental updating algorithm of approximate set based on the dominance relationship in ordered information system was proposed by $\mathrm{Li}$ et al. [11]. Considering the data analysis of dual universes, Hu et al. studied the dynamic updating approximations [12]. Luo et al. explored an efficient updating approach of probabilistic rough set with incremental objects [13].

Updating data with the variation of attributes. Hu et al. proposed two matrix-based incremental strategies, which can dynamically update the upper and lower approximations of each decision class of multi-granulation rough set based on dominance-based relationship [14]. Lang et al. studied the incremental mechanism of updating the upper and lower approximations with the change of attributes in the dynamic covering information system [15]. Based on the definition of relation matrix, diagonal matrix and cut matrix in multi-granulation rough set, Hu et al. put forward the matrix representation of upper and lower approximations in optimistic and pessimistic multi-granulation rough set, and then gave the dynamic updated approximations based on matrix [16]. Cheng proposed an efficient incremental algorithm for rough fuzzy approximations and applied it to attribute reduction [17]. Zhang et al. proposed the concept of basic vector induced by relational matrix for set-valued information system and then put forward the static and dynamic methods of rough approximations for set-valued information systems [18]. Li further put forward a calculated method of approximations based on the dominant matrix for dominance rough set [19]. Yang et al. proposed an approach for updating dynamic approximations in multi-granulation rough sets variation of granular structures [20].

Updating data with the variation of attribute values, aiming at the dynamic updating of attribute values in information system, Chen et al. first defined the concept of attribute values coarsening and refining, and designed an efficient dynamic algorithm of approximations [21], further discussed the incremental updating of approximations based on the dominance relationship [22]. Wang et al. put forward an incremental algorithm of attribute reduction based on the rough set theory [23]. An incremental algorithm of updating decision rules was proposed for inconsistent decision table by Chen et al. [24]. Li et al. proposed a fast method of approximations by using matrix operation when attribute values are updated dynamically in ordered information system [25]. Luo et al.come up with a fast algorithm for computing rough approximations in set-valued decision systems [26]. Zeng et al. proposed a dynamical updating method of fuzzy rough approximations for hybrid data under the variation of attribute values [27]. Hu et al. put forward a dynamic algorithm of updating approximations in multi-granulation rough sets by using approximations's monotonicity directly [28]. 
Most of the existing dynamic updated studies occurred under the situation of single granule, but as demanded, there is a situation of multiple granules that we should explore its dynamic updating, i.e., multi-granulation rough set. Professor Qian Yuhua first proposed the multi-granulation rough set [29], optimistic multi-granulation rough set and pessimistic multi-granulation rough set were studied, approximating the concept and granulating the universe by combining or intersecting attributes. multi-granulation rough set is very important, it can be applied to multiple contexts and produce multiple types of multi-granulation rough set, such as Incomplete multi-granulation rough set [30], Neighborhood-based multi-granulation rough sets [31], Multi-granulation decision-theoretic rough sets [32], Intuitionistic fuzzy multi-granulation rough sets [33], Variable precision multi-granulation decision-theoretic fuzzy rough sets [34], Local multi-granulation decision-theoretic rough sets [35], Generalized multi-granulation rough sets [36], and so on.

There are few studies on the multi-granulation rough set about dynamic updating, and when adding or deleting objects, there is no studies on multi-granulation rough set model. Thus this paper mainly focuses on this problem. For dynamic multi-granulation rough set models, the approximations will change with the change of objects in the universe, and sometimes objects of the target concept will change from the change of the universe, and it makes us not able to seek approximations by using monotonicity directly. Given this situation, two dynamic algorithms are given. We first explore the relationships between the original approximations and updating approximations and propose the dynamic process of updating the lower and upper approximations when adding or deleting objects, and then the corresponding dynamic algorithms are proposed in the multi-granulation rough set. Finally, the effectiveness of two algorithms is verified by experiments.

The rest of this paper is organized as follows. The basic concepts and properties of rough sets and multigranulation rough sets are briefly introduced in Section 2. In section 3, the methods of updating the lower and upper approximations are proposed when adding or deleting objects. Then the static algorithm and two dynamic algorithms are given in Section 4. In section 5, we verify the effectiveness of the proposed dynamic algorithm experimentally. Finally, Section 6 concludes this paper.

\section{Preliminaries}

In this section, we first review some concepts and propositions of rough sets and multi-granulation rough sets.

\subsection{Rough sets}

Definition 1. [1] Give an information system $I S=\langle U, A T, V, f\rangle$, where $U=\left\{x_{1}, x_{2}, \ldots, x_{n}\right\}$ is a non-empty finite set of objects called universe; $A T$ is a non-empty finite set of attributes, $a \in A T$ is called an attribute; $V=\bigcup_{a \in A T} V_{a}$ is a set of attributes values; where $V_{a}$ is a non-empty set of values of attribute $a \in A T$, called the domain of $a$; $f: U \times A T \rightarrow V$ is an information function that maps an object in $U$ to exactly one value in $V_{a}$ such that $\forall a \in A T, x_{i} \in$ $U, f\left(x_{i}, a\right) \in V_{a}$.

Definition 2. [1] Give an information system $I S=\langle U, A T, V, f\rangle$, Each subset of attributes $B \subseteq A T$ determines an 
indiscernibility relation $R_{B}$ as follows:

$$
R_{B}=\left\{\left(x_{i}, y_{i}\right) \in U \times U: f\left(x_{i}, a\right)=f\left(y_{i}, a\right), \forall a \in B\right\} .
$$

$R_{B}$ is an equivalence relation on $U$. The equivalence relation $R_{B}$ partitions the universe $U$ into a family of disjoint subsets called equivalence classes, the equivalence class including $x_{i}$ with respect to $B$ is denoted as $\left[x_{i}\right]_{B}=\{y \in U$ : $\left.\left(x_{i}, y\right) \in R_{B}\right\}$.

Definition 3. [1] Give an information system $I S=\langle U, A T, V, f\rangle$, For any $X \subseteq U, R$ is an equivalence relation, two subsets of objects, called lower and upper approximations of $X$ with respect to $R$, are difened as:

$$
\begin{aligned}
& \underline{R}(X)=\left\{x_{i} \in U:\left[x_{i}\right]_{R} \subseteq X\right\}, \\
& \bar{R}(X)=\left\{x_{i} \in U:\left[x_{i}\right]_{R} \cap X \neq \emptyset\right\} .
\end{aligned}
$$

where $\left[x_{i}\right]_{R}=\left\{y \in U \mid\left(x_{i}, y\right) \in R\right\}$ is the $R$-equivalence class containing $x_{i}$.

If $\underline{R}(X)=\bar{R}(X)$, we say that $X$ is a definable set; Otherwise, $X$ is a rough set.

2.2. Multi-granulation rough sets(MGRS)

Definition 4. [29] Give an information system $I S=\langle U, A T, V, f\rangle, A_{1}, A_{2}, \ldots, A_{m} \subseteq A T$, and $\forall X \subseteq U$, the optimistic multi-granulation lower and upper approximations of the set $X$ with respect to $A_{1}, A_{2}, \ldots, A_{m}$ are denoted by $\frac{\sum_{k=1}^{m} A_{k}{ }^{O}}{m}(X)$ and ${\overline{\sum_{k=1}^{m} A_{k}}}^{O}(X)$, respectively, where

$$
\begin{aligned}
& \sum_{k=1}^{m} A_{k}{ }^{O}(X)=\left\{x_{i} \in U:\left[x_{i}\right]_{A_{1}} \subseteq X \vee\left[x_{i}\right]_{A_{2}} \subseteq X \vee \ldots \vee\left[x_{i}\right]_{A_{m}} \subseteq X\right\}, \\
& \overline{\sum_{k=1}^{m} A_{k}}(X)=\sim \sum_{k=1}^{m} A_{k}{ }^{o}(\sim X) .
\end{aligned}
$$

Proposition 1. [29] Give an information system $I S=\langle U, A T, V, f\rangle, A_{1}, A_{2}, \ldots, A_{m} \subseteq A T$, and $\forall X \subseteq U$, we have the following properties:

$$
{\overline{\sum_{k=1}^{m} A_{k}}}^{O}(X)=\left\{x_{i} \in U:\left[x_{i}\right]_{A_{1}} \cap X \neq \emptyset \wedge\left[x_{i}\right]_{A_{2}} \cap X \neq \emptyset \wedge \ldots \wedge\left[x_{i}\right]_{A_{m}} \cap X \neq \emptyset\right\} .
$$

Proposition 2. [29] Give an information system $I S=\langle U, A T, V, f\rangle, A_{1}, A_{2}, \ldots, A_{m} \subseteq A T$, and $X, Y \subseteq U$. If $X \subseteq Y$, the following properties hold:
(1) $\sum_{k=1}^{m} A_{k}{ }^{O}(X) \subseteq \sum^{m} A_{k}^{O}(Y)$,

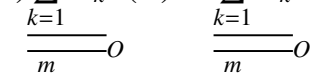
(2) $\sum_{k=1}^{m} A_{k}(X) \subseteq \sum_{k=1}^{m} A_{k}(Y)$.

Definition 5. [29] Give an information system $I S=\langle U, A T, V, f\rangle, A_{1}, A_{2}, \ldots, A_{m} \subseteq A T$, and $\forall X \subseteq U$, the pessimistic multi-granulation lower and upper approximations of the set $X$ with respect to $A_{1}, A_{2}, \ldots, A_{m}$ are denoted by $\frac{\sum_{k=1}^{m} A_{k}{ }^{P}}{m}(X)$ and ${\overline{\sum_{k=1}^{m} A_{k}}}^{P}(X)$, respectively, where

$$
\begin{aligned}
& \sum_{k=1}^{P} A_{k} \\
& {\overline{\sum_{k=1}^{m} A_{k}}}^{P}(X)=\left\{x_{i} \in U:\left[x_{i}\right]_{A_{1}} \subseteq X \wedge\left[x_{i}\right]_{A_{2}} \subseteq X \wedge \ldots \wedge\left[x_{i}\right]_{A_{m}} \subseteq X\right\}, \\
& \sum_{k=1}^{m} A_{k} P(\sim X) .
\end{aligned}
$$

Proposition 3. [29] Give an information system $I S=\langle U, A T, V, f\rangle, A_{1}, A_{2}, \ldots, A_{m} \subseteq A T$, and $\forall X \subseteq U$, we have the following properties: 
${\overline{\sum_{k=1}^{m} A_{k}}}^{P}(X)=\left\{x_{i} \in U:\left[x_{i}\right]_{A_{1}} \cap X \neq \emptyset \vee\left[x_{i}\right]_{A_{2}} \cap X \neq \emptyset \vee \ldots \vee\left[x_{i}\right]_{A_{m}} \cap X \neq \emptyset\right\}$

Proposition 4. [29] Give an information system $I S=\langle U, A T, V, f\rangle, A_{1}, A_{2}, \ldots, A_{m} \subseteq A T$, and $X, Y \subseteq U$. If $X \subseteq Y$, the following properties hold:

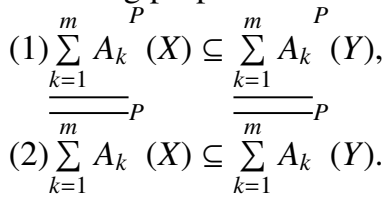

Table 1: An information system

\begin{tabular}{ccccc}
\hline$U$ & $a_{1}$ & $a_{2}$ & $a_{3}$ & $a_{4}$ \\
\hline$x_{1}$ & 1 & 1 & 1 & 3 \\
$x_{2}$ & 3 & 1 & 1 & 1 \\
$x_{3}$ & 2 & 1 & 1 & 3 \\
$x_{4}$ & 3 & 2 & 1 & 3 \\
$x_{5}$ & 2 & 3 & 2 & 2 \\
$x_{6}$ & 1 & 2 & 1 & 1 \\
$x_{7}$ & 1 & 3 & 2 & 3 \\
$x_{8}$ & 3 & 3 & 2 & 2 \\
\hline
\end{tabular}

Example 1. Give an information system in Table 1, $U=\left\{x_{1}, x_{2}, x_{3}, x_{4}, x_{5}, x_{6}, x_{7}, x_{8}\right\}, A T=\left\{a_{1}, a_{2}, a_{3}, a_{4}\right\}$, $A_{1}=\left\{a_{1}\right\}, A_{2}=\left\{a_{2}\right\}, A_{3}=\left\{a_{3}\right\}, A_{4}=\left\{a_{4}\right\}, X=\left\{x_{3}, x_{4}, x_{5}, x_{7}, x_{8}\right\}$. According to Definition 4 and Definition 5, we can calculate the optimistic multi-granulation lower and upper approximations, the pessimistic multi-granulation lower and upper approximations of the set $X$ as follows:

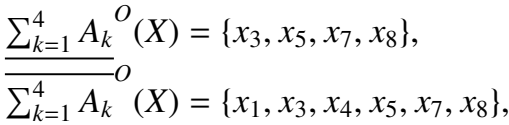

$$
\begin{aligned}
& \sum_{k=1}^{4} A_{k}{ }^{P}(X)=\left\{x_{5}\right\}, \\
& {\overline{\sum_{k=1}^{4} A_{k}}}^{P}(X)=\left\{x_{1}, x_{2}, x_{3}, x_{4}, x_{5}, x_{6}, x_{7}, x_{8}\right\} \text {. }
\end{aligned}
$$

\section{Updating multi-granulation rough approximations with increasing or decreasing of objects}

3.1 Updating multi-granulation rough approximations while increasing objects

In this subsection, we define the concept of optimistic multi-granulation rough set and pessimistic multi-granulation rough set in the new information system after adding objects and then discuss the relationship between the original approximations and updated approximations.

Definition 6. Give an information system $I S=\langle U, A T, V, f\rangle, A_{1}, A_{2}, \ldots, A_{m} \subseteq A T, U=\left\{x_{1}, x_{2}, \ldots, x_{n}\right\}, X \subseteq U$, adding $n^{\prime}$ new objects to $U$, assume that the new universe is $U^{\prime}=U \cup U^{+}$, where $U^{+}=\left\{x_{n+1}, x_{n+2}, \ldots, x_{n+n^{\prime}}\right\}$. 
$\forall X^{\prime} \subseteq U^{\prime}$, the optimistic multi-granulation lower and upper approximation of $X^{\prime}$ in the new universe are denoted by $\underline{\sum_{k=1}^{m} A_{k}^{O \vee}}\left(X^{\prime}\right)$ and ${\overline{\sum_{k=1}^{m} A_{k}}}^{O \vee}\left(X^{\prime}\right)$, respectively,

$$
\begin{aligned}
& \frac{\sum_{k=1}^{m} A_{k}}{O \vee}\left(X^{\prime}\right)=\left\{x_{i} \in U^{\prime}:\left[x_{i}\right]_{A_{1}}^{\vee} \subseteq X^{\prime} \vee\left[x_{i}\right]_{A_{2}}^{\vee} \subseteq X^{\prime} \vee \ldots \vee\left[x_{i}\right]_{A_{m}}^{\vee} \subseteq X^{\prime}\right\}, \\
& \overline{\sum_{k=1}^{m} A_{k}}\left(X^{\prime}\right)=\left\{x_{i} \in U^{\prime}:\left[x_{i}\right]_{A_{1}}^{\vee} \cap X^{\prime} \neq \emptyset \wedge\left[x_{i}\right]_{A_{2}}^{\vee} \cap X^{\prime} \neq \emptyset \wedge \ldots \wedge\left[x_{i}\right]_{A_{m}}^{\vee} \cap X^{\prime} \neq \emptyset\right\} .
\end{aligned}
$$

where $\left[x_{i}\right]_{A_{k}}^{\vee}$ is the equivalence class including $x_{i}$ with respect to a granular $A_{k}$ in the new universe.

Definition 7. Give an information system $I S=\langle U, A T, V, f\rangle, A_{1}, A_{2}, \ldots, A_{m} \subseteq A T, U=\left\{x_{1}, x_{2}, \ldots, x_{n}\right\}, X \subseteq U$, adding $n^{\prime}$ new objects to $U$, assume that the new universe is $U^{\prime}=U \cup U^{+}$, where $U^{+}=\left\{x_{n+1}, x_{n+2}, \ldots, x_{n+n^{\prime}}\right\}$. $\forall X^{\prime} \subseteq U^{\prime}$, the pessimistic multi-granulation lower and upper approximation of $X^{\prime}$ in the new universe are denoted by $\underline{\sum_{k=1}^{m} A_{k}^{P \vee}}\left(X^{\prime}\right)$ and ${\overline{\sum_{k=1}^{m} A_{k}}}^{P \vee}\left(X^{\prime}\right)$, respectively,

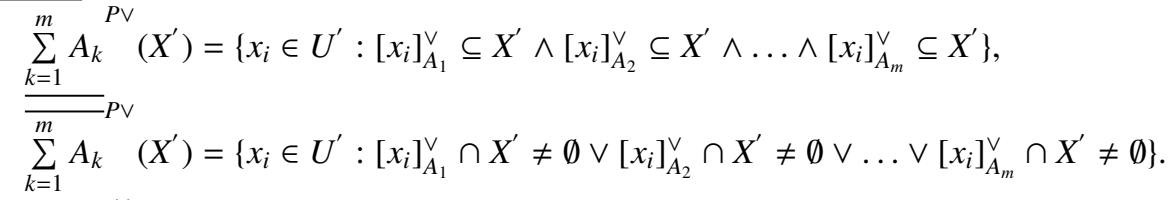
where $\left[x_{i}\right]_{A_{k}}^{\vee}$ is the equivalence class including $x_{i}$ with respect to a granular $A_{k}$ in the new universe.

Theorem 1. Give an information system $I S=\langle U, A T, V, f\rangle, A_{1}, A_{2}, \ldots, A_{m} \subseteq A T$, and $\forall X \subseteq U$, the following results hold:

(1) $\frac{\sum_{k=1}^{m} A_{k}^{O}}{\overline{\sum_{k=1}^{m} A_{k}}} O(X) \supseteq \underline{\underline{\sum_{k=1}^{m} A_{k}^{O}}} O \vee(X)$;

(2) ${\overline{\sum_{k=1}^{m} A_{k}}}^{O}(X) \subseteq{\overline{\sum_{k=1}^{m} A_{k}}}^{O \vee}(X)$.

Proof. (1) $\forall x \in{\underline{\sum_{k=1}^{m} A_{k}}}^{O \vee}(X)$, by Definition 6, we have $\exists k \in\{1,2, \ldots, m\},[x]_{A_{k}}^{\vee} \subseteq X$. When adding objects, by Definition 6, we have $[x]_{A_{k}} \subseteq[x]_{A_{k}}^{\vee}$. Thus, $\exists k \in\{1,2, \ldots, m\},[x]_{A_{k}} \subseteq X$. According to Definition 4, $x \in \sum_{k=1}^{m} A_{k}^{O}(X)$. Therefore, $\sum_{k=1}^{m} A_{k}^{O}(X) \supseteq \sum_{k=1}^{m} A_{k}^{O \vee}(X)$;

(2) $\forall x \in{\overline{\sum_{k=1}^{m} A_{k}}}^{O}(X)$, by Proposition 1, we have $\forall k \in\{1,2, \ldots, m\},[x]_{A_{k}} \cap X \neq \emptyset$. When adding objects, by Definition 6, we have $[x]_{A_{k}} \subseteq[x]_{A_{k}}^{\vee}$. Thus, $\forall k \in\{1,2, \ldots, m\},[x]_{A_{k}}^{\vee} \cap X \neq \emptyset$. According to Definition $6, x \in$ ${\overline{\sum_{k=1}^{m} A_{k}}}^{O \vee}(X)$. Therefore, ${\overline{\sum_{k=1}^{m} A_{k}}}^{O}(X) \subseteq{\overline{\sum_{k=1}^{m} A_{k}}}^{O \vee}(X)$.

Theorem 2. Give an information system $I S=\langle U, A T, V, f\rangle, A_{1}, A_{2}, \ldots, A_{m} \subseteq A T$, let $X^{+} \subseteq U^{+}$, and $\forall X \subseteq U$, $X^{\prime} \subseteq U^{\prime}$, if $X^{\prime}=X \cup X^{+}$, the following results hold:
(1) $\underline{\sum}_{k=1}^{m} A_{k}^{O \vee}(X)=\underline{\sum}_{k=1}^{m} A_{k}^{O}(X)-\Delta H_{1}$, $\Delta H_{1}=\left\{x_{i} \in \sum_{k=1}^{m} A_{k}^{O}(X):\left[x_{i}\right]_{A_{k}}^{\vee} \nsubseteq X, \forall k \in\{1,2, \ldots, m\}\right\} ;$
(2) ${\underline{\sum_{k=1}^{m} A_{k}^{O \vee}}}^{O \vee}\left(X^{\prime}\right)=\underline{\sum_{k=1}^{m} A_{k}}{ }^{O \vee}(X) \cup \Delta H_{2}$,
$\Delta H_{2}=\bigcup\left\{\left[x_{j}\right]_{A_{k}}^{\vee}:\left[x_{j}\right]_{A_{k}}^{\vee} \subseteq X^{\prime}, x_{j} \in X^{+}\right\} ;$
(3) ${\overline{\sum_{k=1}^{m} A_{k}}}^{O \vee}\left(X^{\prime}\right)={\overline{\sum_{k=1}^{m} A_{k}}}^{O}(X) \cup \Delta H_{3}$, $\Delta H_{3}=\left\{x_{i} \in U^{\prime}-{\overline{\sum_{k=1}^{m} A_{k}}}^{O}(X):\left[x_{i}\right]_{A_{k}}^{\vee} \cap X^{\prime} \neq \emptyset, \forall k \in\{1,2, \ldots, m\}\right\}$.

Proof. (1) $\forall x \in \sum_{k=1}^{m} A_{k}^{O}(X)$, by Definition 4, we have $\exists k \in\{1,2, \ldots, m\},[x]_{A_{k}} \subseteq X$. If $\exists k \in\{1,2, \ldots, m\}$, $[x]_{A_{k}}^{\vee} \subseteq X$, then $x \in{\overline{\sum_{k=1}^{m} A_{k}}}^{O \vee}(X)$; If $\forall k \in\{1,2, \ldots, m\},[x]_{A_{k}}^{\vee} \nsubseteq X$, then $x \in \Delta H_{1}$. Therefore, ${\underline{\sum_{k=1}^{m} A_{k}}}^{O \vee}(X)=$

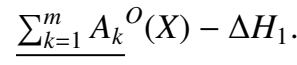


(2) “ $\Rightarrow$ ” $\forall x \in \sum_{k=1}^{m} A_{k}^{O \vee}\left(X^{\prime}\right)$, by Definition 6, we have $\exists k \in\{1,2, \ldots, m\},[x]_{A_{k}}^{\vee} \subseteq X^{\prime}$. If $\exists k \in\{1,2, \ldots, m\}$, $[x]_{A_{k}}^{\vee} \subseteq X$, then $x \in{\underline{\sum_{k=1}^{m} A_{k}}}^{O \vee}(X)$. Otherwise, $\exists x_{j} \in X^{+},[x]_{A_{k}}^{\vee}=\left[x_{j}\right]_{A_{k}}^{\vee}$, i.e., $x \in\left[x_{j}\right]_{A_{k}}^{\vee}$. Therefore, $\sum_{k=1}^{m} A_{k}{ }^{O \vee}\left(X^{\prime}\right) \subseteq$ $\sum_{k=1}^{m} A_{k}^{O \vee}(X) \cup \Delta H_{2}$.

“ $\Leftarrow$ " It is obvious that $\Delta H_{2} \subseteq \underline{\sum_{k=1}^{m} A_{k}{ }^{O \vee}}\left(X^{\prime}\right)$ by Definition 6. By Proposition 2, $\underline{\sum_{k=1}^{m} A_{k}{ }^{O \vee}}(X) \subseteq \underline{\sum_{k=1}^{m} A_{k}{ }^{O \vee}}\left(X^{\prime}\right)$. Therefore, $\sum_{k=1}^{m} A_{k}{ }^{\circ \vee}(X) \cup \Delta H_{2} \subseteq \sum_{k=1}^{m} A_{k}{ }^{O \vee}\left(X^{\prime}\right)$.

In conclusion, $\sum_{k=1}^{m} A_{k}{ }^{O \vee}\left(X^{\prime}\right)={\underline{\sum_{k=1}^{m} A_{k}}}^{O \vee}(X) \cup \Delta H_{2}$.

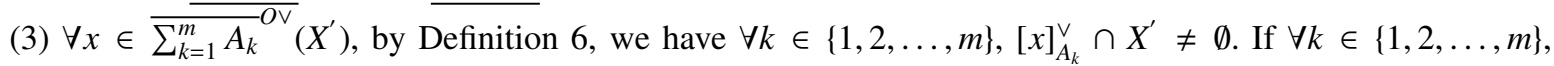
$[x]_{A_{k}} \cap X \neq \emptyset$, then $x \in{\overline{\sum_{k=1}^{m} A_{k}}}^{O}(X)$; Otherwise, $x \notin{\overline{\sum_{k=1}^{m} A_{k}}}^{O}(X)$, i.e., $x \in \Delta H_{3}$. Therefore, ${\overline{\sum_{k=1}^{m} A_{k}}}^{O \vee}\left(X^{\prime}\right)=$ ${\overline{\sum_{k=1}^{m} A_{k}}}^{O}(X) \cup \Delta H_{3}$.

Table 2: An information system after adding objects

\begin{tabular}{ccccc}
\hline$U^{\prime}$ & $a_{1}$ & $a_{2}$ & $a_{3}$ & $a_{4}$ \\
\hline$x_{1}$ & 1 & 1 & 1 & 3 \\
$x_{2}$ & 3 & 1 & 1 & 1 \\
$x_{3}$ & 2 & 1 & 1 & 3 \\
$x_{4}$ & 3 & 2 & 1 & 3 \\
$x_{5}$ & 2 & 3 & 2 & 2 \\
$x_{6}$ & 1 & 2 & 1 & 1 \\
$x_{7}$ & 1 & 3 & 2 & 3 \\
$x_{8}$ & 3 & 3 & 2 & 2 \\
$x_{9}$ & 1 & 1 & 2 & 2 \\
$x_{10}$ & 3 & 3 & 1 & 1 \\
\hline
\end{tabular}

Example 2. (Continuation of Example 1) The Table 2 is the expansion of Table 1, let $U^{+}=\left\{x_{9}, x_{10}\right\}, X^{+}=\left\{x_{9}\right\}$, $X^{\prime}=X \cup X^{+}$. In the new universe, according to Theorem 2 and the results of Example 1, we can calculate the optimistic lower approximation and the upper approximation of $X^{\prime}$ as follows:

$U^{\prime}=U \cup U^{+}=\left\{x_{1}, x_{2}, x_{3}, x_{4}, x_{5}, x_{6}, x_{7}, x_{8}, x_{9}, x_{10}\right\}$,

$X^{\prime}=X \cup X^{+}=\left\{x_{3}, x_{4}, x_{5}, x_{7}, x_{8}, x_{9}\right\}$

(1) $\sum_{k=1}^{4} A_{k}^{O}(X)=\left\{x_{3}, x_{5}, x_{7}, x_{8}\right\}$,

$\left[x_{3}\right]_{A_{1}}^{\vee} \subseteq X, x_{3} \notin \Delta H_{1}$;

$\left[x_{5}\right]_{A_{1}}^{\vee} \subseteq X,\left[x_{5}\right]_{A_{2}}^{\vee} \nsubseteq X, x_{5} \notin \Delta H_{1}$;

$\left[x_{7}\right]_{A_{1}}^{\vee} \nsubseteq X,\left[x_{7}\right]_{A_{2}}^{\vee} \nsubseteq X,\left[x_{7}\right]_{A_{3}}^{\vee} \nsubseteq X,\left[x_{7}\right]_{A_{4}}^{\vee} \nsubseteq X, x_{7} \in \Delta H_{1} ;$

$\left[x_{8}\right]_{A_{1}}^{\vee} \nsubseteq X,\left[x_{8}\right]_{A_{2}}^{\vee} \nsubseteq X,\left[x_{8}\right]_{A_{3}}^{\vee} \nsubseteq X,\left[x_{8}\right]_{A_{4}}^{\vee} \nsubseteq X, x_{8} \in \Delta H_{1} ;$

Then, $\Delta H_{1}=\left\{x_{7}, x_{8}\right\}, \sum_{k=1}^{4} A_{k}^{O \vee}(X)=\sum_{k=1}^{4} A_{k}^{o}(X)-\Delta H_{1}=\left\{x_{3}, x_{5}\right\}$.

$\left[x_{9}\right]_{A_{1}}^{\vee} \nsubseteq X^{\prime},\left[x_{9}\right]_{A_{2}}^{\vee} \nsubseteq X^{\prime},\left[x_{9}\right]_{A_{3}}^{\vee} \subseteq X^{\prime},\left[x_{9}\right]_{A_{4}}^{\vee} \subseteq X^{\prime} ;$ 
Therefore, $\Delta H_{2}=\left[x_{9}\right]_{A_{3}}^{\vee} \cup\left[x_{9}\right]_{A_{4}}^{\vee}=\left\{x_{5}, x_{7}, x_{8}, x_{9}\right\}, \sum_{k=1}^{4} A_{k}^{O \vee}\left(X^{\prime}\right)=\sum_{k=1}^{4} A_{k}^{O \vee}(X) \cup \Delta H_{2}=\left\{x_{3}, x_{5}, x_{7}, x_{8}, x_{9}\right\}$.

(2) ${\overline{\sum_{k=1}^{4} A_{k}}}^{O}(X)=\left\{x_{1}, x_{3}, x_{4}, x_{5}, x_{7}, x_{8}\right\}$,

$\left[x_{2}\right]_{A_{1}}^{\vee} \cap X^{\prime} \neq \emptyset,\left[x_{2}\right]_{A_{2}}^{\vee} \cap X^{\prime} \neq \emptyset,\left[x_{2}\right]_{A_{3}}^{\vee} \cap X^{\prime} \neq \emptyset,\left[x_{2}\right]_{A_{4}}^{\vee} \cap X^{\prime}=\emptyset, x_{2} \notin \Delta H_{3} ;$

$\left[x_{6}\right]_{A_{1}}^{\vee} \cap X^{\prime} \neq \emptyset,\left[x_{6}\right]_{A_{2}}^{\vee} \cap X^{\prime} \neq \emptyset,\left[x_{6}\right]_{A_{3}}^{\vee} \cap X^{\prime} \neq \emptyset,\left[x_{6}\right]_{A_{4}}^{\vee} \cap X^{\prime}=\emptyset, x_{6} \notin \Delta H_{3} ;$

$\left[x_{9}\right]_{A_{1}}^{\vee} \cap X^{\prime} \neq \emptyset,\left[x_{9}\right]_{A_{2}}^{\vee} \cap X^{\prime} \neq \emptyset,\left[x_{9}\right]_{A_{3}}^{\vee} \cap X^{\prime} \neq \emptyset,\left[x_{9}\right]_{A_{4}}^{\vee} \cap X^{\prime} \neq \emptyset, x_{9} \in \Delta H_{3} ;$

$\left[x_{10}\right]_{A_{1}}^{\vee} \cap X^{\prime} \neq \emptyset,\left[x_{10}\right]_{A_{2}}^{\vee} \cap X^{\prime} \neq \emptyset,\left[x_{10}\right]_{A_{3}}^{\vee} \cap X^{\prime} \neq \emptyset,\left[x_{10}\right]_{A_{4}}^{\vee} \cap X^{\prime}=\emptyset, x_{10} \notin \Delta H_{3} ;$

Therefore, $\Delta H_{3}=\left\{x_{9}\right\},{\overline{\sum_{k=1}^{4} A_{k}}}^{O \vee}\left(X^{\prime}\right)={\overline{\sum_{k=1}^{4} A_{k}}}^{O}(X) \cup \Delta H_{3}=\left\{x_{1}, x_{3}, x_{4}, x_{5}, x_{7}, x_{8}, x_{9}\right\}$.

Theorem 3. Give an information system $I S=\langle U, A T, V, f\rangle, A_{1}, A_{2}, \ldots, A_{m} \subseteq A T$, and $\forall X \subseteq U$, the following results hold:

(1) $\sum_{k=1}^{m} A_{k}^{P}(X) \supseteq \sum_{k=1}^{m} A_{k}^{P \vee}(X)$;

(2) ${\overline{\overline{\sum_{k=1}^{m} A_{k}}}}^{P}(X) \subseteq{\overline{\overline{\sum_{k=1}^{m} A_{k}}}}^{P \vee}(X)$.

Proof. (1) $\forall x \in{\underline{\sum_{k=1}^{m}}}_{A_{k}}^{P \vee}(X)$, by Definition 7, we have $\forall k \in\{1,2, \ldots, m\},[x]_{A_{k}}^{\vee} \subseteq X$. When adding objects, by Definition 7, we have $[x]_{A_{k}} \subseteq[x]_{A_{k}}^{\vee}$. Thus, $\forall k \in\{1,2, \ldots, m\},[x]_{A_{k}} \subseteq X$. According to Definition 5, $x \in \underline{\sum_{k=1}^{m} A_{k}^{P}}(X)$.

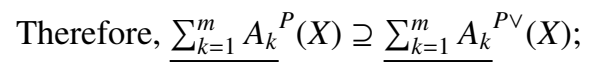

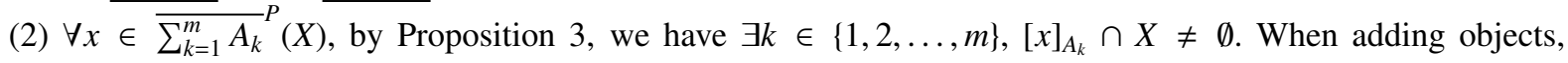
by Definition 7, we have $[x]_{A_{k}} \subseteq[x]_{A_{k}}^{\vee}$. Thus, $\exists k \in\{1,2, \ldots, m\},[x]_{A_{k}}^{\vee} \cap X \neq \emptyset$. According to Definition $7, x \in$ ${\overline{\sum_{k=1}^{m} A_{k}}}^{P \vee}(X)$. Therefore, ${\overline{\sum_{k=1}^{m} A_{k}}}^{P}(X) \subseteq{\overline{\sum_{k=1}^{m} A_{k}}}^{P \vee}(X)$.

Theorem 4. Give an information system $I S=\langle U, A T, V, f\rangle, A_{1}, A_{2}, \ldots, A_{m} \subseteq A T$, let $X^{+} \subseteq U^{+}$, and $\forall X \subseteq U$, $X^{\prime} \subseteq U^{\prime}$, if $X^{\prime}=X \cup X^{+}$, the following results hold:
(1) ${\underline{\sum_{k=1}^{m} A_{k}^{P \vee}}}^{P H_{4}}(X)=\underline{\sum_{k=1}^{m} A_{k}}{ }^{P}(X)-\Delta H_{4}$, $\Delta H_{4}=\left\{x_{i} \in \sum_{k=1}^{m} A_{k}^{P}(X):\left[x_{i}\right]_{A_{k}}^{\vee} \nsubseteq X, \exists k \in\{1,2, \ldots, m\}\right\} ;$
(2) $\sum_{k=1}^{m} A_{k}^{P \vee}\left(X^{\prime}\right)=\underline{\sum_{k=1}^{m}} A_{k}^{P \vee}(X) \cup \Delta H_{5}$, $\Delta H_{5}=\left\{x_{j} \in U^{\prime}-\sum_{k=1}^{m} A_{k}^{P \vee}(X):\left[x_{j}\right]_{A_{k}}^{\vee} \subseteq X^{\prime}, \forall k \in\{1,2, \ldots, m\}\right\} ;$
(3) ${\overline{\sum_{k=1}^{m} A_{k}}}^{P \vee}\left(X^{\prime}\right)={\overline{\sum_{k=1}^{m} A_{k}}}^{P}(X) \cup \Delta H_{6}$, $\Delta H_{6}=\bigcup\left\{\left[x_{i}\right]_{A_{k}}^{\vee}:\left[x_{i}\right]_{A_{k}}^{\vee} \cap X^{\prime} \neq \emptyset, x_{i} \in U^{+}\right\}$.

Proof. (1) $\forall x \in \sum_{k=1}^{m} A_{k}^{P}(X)$, by Definition 5, we have $\forall k \in\{1,2, \ldots, m\},[x]_{A_{k}} \subseteq X$. If $\forall k \in\{1,2, \ldots, m\}$, $[x]_{A_{k}}^{\vee} \subseteq X$, then $x \in{\underline{\sum_{k=1}^{m} A_{k}^{P}}}^{\vee \vee}(X)$; If $\exists k \in\{1,2, \ldots, m\},[x]_{A_{k}}^{\vee} \nsubseteq X$, then $x \in \Delta H_{4}$. Therefore, $\underline{\sum}_{k=1}^{m} A_{k}^{P \vee}(X)=$ $\underline{\sum_{k=1}^{m} A_{k}^{P}}(X)-\Delta H_{4}$.

(2) $\forall x \in \underline{\sum_{k=1}^{m} A_{k}^{P \vee}}\left(X^{\prime}\right)$, by Definition 7, we have $\forall k \in\{1,2, \ldots, m\},[x]_{A_{k}}^{\vee} \subseteq X^{\prime}$. If $\forall k \in\{1,2, \ldots, m\},[x]_{A_{k}}^{\vee} \subseteq X$, then $x \in \underline{\sum_{k=1}^{m}} \overline{A_{k}{ }^{P \vee}}(X)$. If $\exists k \in\{1,2, \ldots, m\},[x]_{A_{k}}^{\vee} \nsubseteq X$, then $x \in \Delta H_{5}$. Therefore, ${\underline{\sum_{k=1}^{m}}}_{A_{k}}^{P \vee}\left(X^{\prime}\right)={\underline{\sum_{k=1}^{m} A_{k}}}^{P \vee}(X) \cup$ $\Delta H_{5}$.

(3) “ $\Rightarrow ” \forall x \in{\overline{\sum_{k=1}^{m} A_{k}}}^{P \vee}\left(X^{\prime}\right)$, by Definition 7, we have $\exists k \in\{1,2, \ldots, m\},[x]_{A_{k}}^{\vee} \cap X^{\prime} \neq \emptyset$. If $\exists k \in\{1,2, \ldots, m\}$, $[x]_{A_{k}} \cap X \neq \emptyset$, then $x \in{\overline{\sum_{k=1}^{m} A_{k}}}^{P}(X)$; Otherwise, $\exists x_{i} \in U^{+},[x]_{A_{k}}^{\vee}=\left[x_{i}\right]_{A_{k}}^{\vee}$, i.e., $x \in[x]_{A_{k}}^{\vee}=\left[x_{i}\right]_{A_{k}}^{\vee}$. Therefore, ${\overline{\sum_{k=1}^{m} A_{k}}}^{P \vee}\left(X^{\prime}\right) \subseteq{\overline{\sum_{k=1}^{m} A_{k}}}^{P}(X) \cup \Delta H_{6}$. 
“ $\Leftarrow "$ It is obvious that $\Delta H_{6} \subseteq{\overline{\sum_{k=1}^{m} A_{k}}}^{P \vee}\left(X^{\prime}\right)$ by Definition 7. By Theorem 3 and Proposition $4,{\overline{\sum_{k=1}^{m} A_{k}}}^{P}(X) \subseteq$ ${\overline{\sum_{k=1}^{m} A_{k}}}^{P \vee}(X) \subseteq{\overline{\sum_{k=1}^{m} A_{k}}}^{P \vee}\left(X^{\prime}\right)$. Therefore, ${\overline{\sum_{k=1}^{m} A_{k}}}^{P}(X) \cup \Delta H_{6} \subseteq{\overline{\sum_{k=1}^{m} A_{k}}}^{P \vee}\left(X^{\prime}\right)$.

In conclusion, ${\overline{\sum_{k=1}^{m} A_{k}}}^{P \vee}\left(X^{\prime}\right)={\overline{\sum_{k=1}^{m} A_{k}}}^{P}(X) \cup \Delta H_{6}$.

Example 3. (Continuation of Example 1) The Table 2 is the expansion of Table 1, let $U^{+}=\left\{x_{9}, x_{10}\right\}, X^{+}=\left\{x_{9}\right\}$, $X^{\prime}=X \cup X^{+}$. In the new universe, according to Theorem 4 and the results of Example 1, we can calculate the pessimistic lower approximation and the upper approximation of $X^{\prime}$ as follows:

$$
\begin{aligned}
& U^{\prime}=U \cup U^{+}=\left\{x_{1}, x_{2}, x_{3}, x_{4}, x_{5}, x_{6}, x_{7}, x_{8}, x_{9}, x_{10}\right\}, \\
& X^{\prime}=X \cup X^{+}=\left\{x_{3}, x_{4}, x_{5}, x_{7}, x_{8}, x_{9}\right\} ;
\end{aligned}
$$

(1) $\sum_{k=1}^{4} A_{k}^{P}(X)=\left\{x_{5}\right\}$,

$\left[x_{5}\right]_{A_{1}}^{\vee} \subseteq X,\left[x_{5}\right]_{A_{2}}^{\vee} \nsubseteq X, x_{5} \in \Delta H_{4}$;

Then, $\Delta H_{4}=\left\{x_{5}\right\}, \underline{\sum_{k=1}^{4} A_{k}{ }^{P \vee}}(X)=\underline{\sum_{k=1}^{4} A_{k}}{ }^{P}(X)-\Delta H_{4}=\emptyset$.

$\left[x_{1}\right]_{A_{1}}^{\vee} \nsubseteq X^{\prime}, x_{1} \notin \Delta H_{5}$

$\left[x_{2}\right]_{A_{1}}^{\vee} \nsubseteq X^{\prime}, x_{2} \notin \Delta H_{5}$;

$\left[x_{3}\right]_{A_{1}}^{\vee} \subseteq X^{\prime},\left[x_{3}\right]_{A_{2}}^{\vee} \nsubseteq X^{\prime}, x_{3} \notin \Delta H_{5}$

$\left[x_{4}\right]_{A_{1}}^{\vee} \nsubseteq X^{\prime}, x_{4} \notin \Delta H_{5}$

$\left[x_{5}\right]_{A_{1}}^{\vee} \subseteq X^{\prime},\left[x_{5}\right]_{A_{2}}^{\vee} \nsubseteq X^{\prime}, x_{5} \notin \Delta H_{5} ;$

$\left[x_{6}\right]_{A_{1}}^{\vee} \nsubseteq X^{\prime}, x_{6} \notin \Delta H_{5}$;

$\left[x_{7}\right]_{A_{1}}^{\vee} \nsubseteq X^{\prime}, x_{7} \notin \Delta H_{5}$

$\left[x_{8}\right]_{A_{1}}^{\vee} \nsubseteq X^{\prime}, x_{8} \notin \Delta H_{5}$

$\left[x_{9}\right]_{A_{1}}^{\vee} \nsubseteq X^{\prime}, x_{9} \notin \Delta H_{5}$

$\left[x_{10}\right]_{A_{1}}^{\vee} \nsubseteq X^{\prime}, x_{10} \notin \Delta H_{5}$

Therefore, $\Delta H_{5}=\emptyset, \underline{\sum_{k=1}^{4} A_{k}{ }^{P \vee}}\left(X^{\prime}\right)={\underline{\sum_{k=1}^{4} A_{k}}}^{P \vee}(X) \cup \Delta H_{5}=\emptyset$.

(2) ${\overline{\sum_{k=1}^{4} A_{k}}}^{P}(X)=\left\{x_{1}, x_{2}, x_{3}, x_{4}, x_{5}, x_{6}, x_{7}, x_{8}\right\}$,

$\left[x_{9}\right]_{A_{1}}^{\vee} \cap X^{\prime} \neq \emptyset,\left[x_{9}\right]_{A_{2}}^{\vee} \cap X^{\prime} \neq \emptyset,\left[x_{9}\right]_{A_{3}}^{\vee} \cap X^{\prime} \neq \emptyset,\left[x_{9}\right]_{A_{4}}^{\vee} \cap X^{\prime} \neq \emptyset ;$
$\left[x_{10}\right]_{A_{1}}^{\vee} \cap X^{\prime} \neq \emptyset,\left[x_{10}\right]_{A_{2}}^{\vee} \cap X^{\prime} \neq \emptyset,\left[x_{10}\right]_{A_{3}}^{\vee} \cap X^{\prime} \neq \emptyset,\left[x_{10}\right]_{A_{4}}^{\vee} \cap X^{\prime}=\emptyset ;$

Therefore, $\Delta H_{6}=\left[x_{9}\right]_{A_{1}}^{\vee} \cup\left[x_{9}\right]_{A_{2}}^{\vee} \cup\left[x_{9}\right]_{A_{3}}^{\vee} \cup\left[x_{9}\right]_{A_{4}}^{\vee} \cup\left[x_{10}\right]_{A_{1}}^{\vee} \cup\left[x_{10}\right]_{A_{2}}^{\vee} \cup\left[x_{10}\right]_{A_{3}}^{\vee}=\left\{x_{1}, x_{2}, x_{3}, x_{4}, x_{5}, x_{6}, x_{7}, x_{8}, x_{9}, x_{10}\right\}$, ${\overline{\sum_{k=1}^{4} A_{k}}}^{P \vee}\left(X^{\prime}\right)={\overline{\sum_{k=1}^{4} A_{k}}}^{P}(X) \cup \Delta H_{6}=\left\{x_{1}, x_{2}, x_{3}, x_{4}, x_{5}, x_{6}, x_{7}, x_{8}, x_{9}, x_{10}\right\}$.

3.2 Updating multi-granulation rough approximations while decreasing objects

In this subsection, we define the concept of optimistic multi-granulation rough set and pessimistic multi-granulation rough set in the new information system after deleting objects and then discuss the relationship between the original approximations and the updated approximations.

Definition 8. Give an information system $I S=\langle U, A T, V, f\rangle, A_{1}, A_{2}, \ldots, A_{m} \subseteq A T, U=\left\{x_{1}, x_{2}, \ldots, x_{n}\right\}, X \subseteq U$, deleting $n^{\prime}$ objects from $U$, assume that the new universe is $U^{\prime}=U-U^{-}$, where $U^{-}$is the set that $n^{\prime}$ objects was deleted from $U . \forall X^{\prime} \subseteq U^{\prime}$, the optimistic multi-granulation lower and upper approximation of $X^{\prime}$ in the new universe 
are denoted by $\sum_{k=1}^{m}{A_{k}}^{O \wedge}\left(X^{\prime}\right)$ and ${\overline{\sum_{k=1}^{m} A_{k}}}^{O \wedge}\left(X^{\prime}\right)$, respectively,

$$
\begin{aligned}
& \sum_{k=1}^{m} A_{k}{ }^{\circ \wedge}\left(X^{\prime}\right)=\left\{x_{i} \in U^{\prime}:\left[x_{i}\right]_{A_{1}}^{\wedge} \subseteq X^{\prime} \vee\left[x_{i}\right]_{A_{2}}^{\wedge} \subseteq X^{\prime} \vee \ldots \vee\left[x_{i}\right]_{A_{m}}^{\wedge} \subseteq X^{\prime}\right\}, \\
& \overline{\sum_{k=1}^{m} A_{k}}\left({ }^{\wedge} \quad\left(X^{\prime}\right)=\left\{x_{i} \in U^{\prime}:\left[x_{i}\right]_{A_{1}}^{\wedge} \cap X^{\prime} \neq \emptyset \wedge\left[x_{i}\right]_{A_{2}}^{\wedge} \cap X^{\prime} \neq \emptyset \wedge \ldots \wedge\left[x_{i}\right]_{A_{m}}^{\wedge} \cap X^{\prime} \neq \emptyset\right\} .\right.
\end{aligned}
$$

where $\left[x_{i}\right]_{A_{k}}^{\wedge}$ is the equivalence class including $x_{i}$ with respect to a granular $A_{k}$ in the new universe.

Definition 9. Give an information system $I S=\langle U, A T, V, f\rangle, A_{1}, A_{2}, \ldots, A_{m} \subseteq A T, U=\left\{x_{1}, x_{2}, \ldots, x_{n}\right\}, X \subseteq U$, deleting $n^{\prime}$ objects from $U$, assume that the new universe is $U^{\prime}=U-U^{-}$, where $U^{-}$is the set that $n^{\prime}$ objects was deleted from $U . \forall X^{\prime} \subseteq U^{\prime}$, the pessimistic multi-granulation lower and upper approximation of $X^{\prime}$ in the new universe

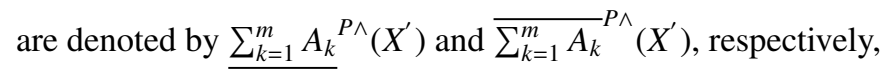

$$
\begin{aligned}
& \sum_{k=1}^{m} A_{k}^{P \wedge}\left(X^{\prime}\right)=\left\{x_{i} \in U^{\prime}:\left[x_{i}\right]_{A_{1}}^{\wedge} \subseteq X^{\prime} \wedge\left[x_{i}\right]_{A_{2}}^{\wedge} \subseteq X^{\prime} \wedge \ldots \wedge\left[x_{i}\right]_{A_{m}}^{\wedge} \subseteq X^{\prime}\right\}, \\
& {\overline{\sum_{k=1}^{m} A_{k}}}^{\wedge}\left(X^{\prime}\right)=\left\{x_{i} \in U^{\prime}:\left[x_{i}\right]_{A_{1}}^{\wedge} \cap X^{\prime} \neq \emptyset \vee\left[x_{i}\right]_{A_{2}}^{\wedge} \cap X^{\prime} \neq \emptyset \vee \ldots \vee\left[x_{i}\right]_{A_{m}}^{\wedge} \cap X^{\prime} \neq \emptyset\right\} .
\end{aligned}
$$

where $\left[x_{i}\right]_{A_{k}}^{\wedge}$ is the equivalence class including $x_{i}$ with respect to a granular $A_{k}$ in the new universe.

Theorem 5. Give an information system $I S=\langle U, A T, V, f\rangle, A_{1}, A_{2}, \ldots, A_{m} \subseteq A T$, and $\forall X^{\prime} \subseteq U^{\prime}$, the following results hold:

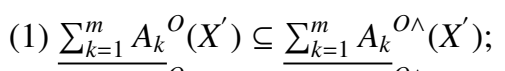

(2) $\overline{\overline{\sum_{k=1}^{m} A_{k}}} O\left(X^{\prime}\right) \supseteq{\overline{\overline{\sum_{k=1}^{m} A_{k}}}}^{O \wedge}\left(X^{\prime}\right)$.

Proof. (1) $\forall x \in \underline{\sum_{k=1}^{m} A_{k}}{ }^{O}\left(X^{\prime}\right)$, by Definition 4, we have $\exists k \in\{1,2, \ldots, m\},[x]_{A_{k}} \subseteq X^{\prime}$. When deleting objects, by

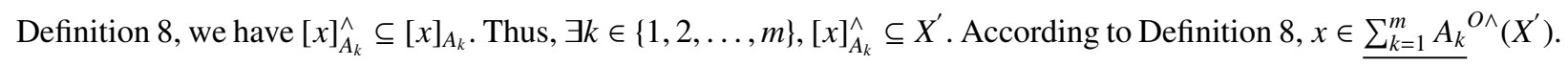
Therefore, $\underline{\sum_{k=1}^{m} A_{k}}{ }^{O}\left(X^{\prime}\right) \subseteq \underline{\sum_{k=1}^{m} A_{k}}{ }^{O \wedge}\left(X^{\prime}\right)$.

(2) $\forall x \in \overline{\sum_{k=1}^{m} A_{k}}{ }^{O \wedge}\left(X^{\prime}\right)$, by Definition 8, we have $\forall k \in\{1,2, \ldots, m\},[x]_{A_{k}}^{\wedge} \cap X^{\prime} \neq \emptyset$. When deleting objects, by Definition 8, we have $[x]_{A_{k}}^{\wedge} \subseteq[x]_{A_{k}}$. Thus, $\forall k \in\{1,2, \ldots, m\},[x]_{A_{k}} \cap X^{\prime} \neq \emptyset$. According to Proposition $1, x \in$ ${\overline{\sum_{k=1}^{m} A_{k}}}^{O}\left(X^{\prime}\right)$. Therefore, ${\overline{\sum_{k=1}^{m} A_{k}}}^{O}\left(X^{\prime}\right) \supseteq{\overline{\sum_{k=1}^{m} A_{k}}}^{O \wedge}\left(X^{\prime}\right)$.

Theorem 6. Give an information system $I S=\langle U, A T, V, f\rangle, A_{1}, A_{2}, \ldots, A_{m} \subseteq A T$, let $X^{-}=U^{-} \cap X$, and $\forall X^{\prime} \subseteq U^{\prime}$, $X \subseteq U$, if $X^{\prime}=X-X^{-}$, the following results hold:

(1) $\underline{\sum_{k=1}^{m} A_{k}{ }^{O}}\left(X^{\prime}\right)=\underline{\sum_{k=1}^{m} A_{k}^{O}}(X)-\Delta H_{7}$,

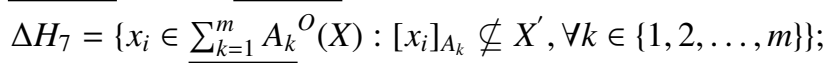

(2) $\underline{\sum_{k=1}^{m} A_{k}}{ }^{O \wedge}\left(X^{\prime}\right)=\underline{\sum_{k=1}^{m} A_{k}}{ }^{O}\left(X^{\prime}\right) \cup \Delta H_{8}$,

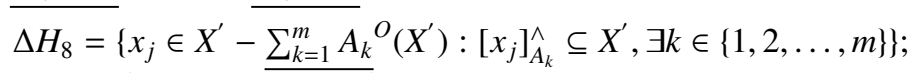

(3) ${\overline{\sum_{k=1}^{m} A_{k}}}^{O \wedge}\left(X^{\prime}\right)={\overline{\sum_{k=1}^{m} A_{k}}}^{O}(X)-U^{-}-\Delta H_{9}$,

$$
\Delta H_{9}=\left\{x_{i} \in{\overline{\sum_{k=1}^{m} A_{k}}}^{O}(X)-U^{-}-X^{\prime}:\left[x_{i}\right]_{A_{k}}^{\wedge} \cap X^{\prime}=\emptyset, \exists k \in\{1,2, \ldots, m\}\right\} .
$$

Proof. (1) $\forall x \in \underline{\sum_{k=1}^{m} A_{k}}{ }^{O}(X)$, by Definition 4, we have $\exists k \in\{1,2, \ldots, m\},[x]_{A_{k}} \subseteq X$. If $\exists k \in\{1,2, \ldots, m\}$, $[x]_{A_{k}} \subseteq X^{\prime}$, then $x \in \underline{\sum_{k=1}^{m} A_{k}}{ }^{O}\left(X^{\prime}\right) ;$ If $\forall k \in\{1,2, \ldots, m\},[x]_{A_{k}} \nsubseteq X^{\prime}$, then $x \in \Delta H_{7}$. Therefore, $\underline{\sum_{k=1}^{m} A_{k}^{O}}\left(X^{\prime}\right)=$ $\underline{\sum_{k=1}^{m} A_{k}^{O}}(X)-\Delta H_{7}$. 
(2) $\forall x \in \underline{\sum_{k=1}^{m} A_{k}^{O \wedge}}\left(X^{\prime}\right)$, by Definition 8, we have $\exists k \in\{1,2, \ldots, m\},[x]_{A_{k}}^{\wedge} \subseteq X^{\prime}$. If $\exists k \in\{1,2, \ldots, m\},[x]_{A_{k}} \subseteq X^{\prime}$,

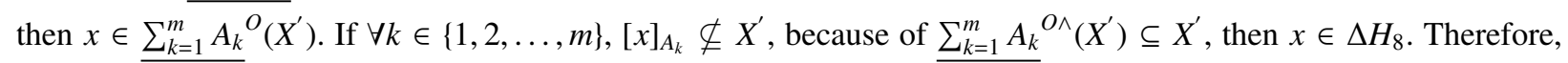

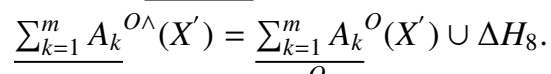

(3) $\forall x \in{\overline{\sum_{k=1}^{m} A_{k}}}^{O}(X)-U^{-}$, by Proposition 1 , we have $\forall k \in\{1,2, \ldots, m\},[x]_{A_{k}} \cap X \neq \emptyset$. If $\forall k \in\{1,2, \ldots, m\}$, $[x]_{A_{k}}^{\wedge} \cap X^{\prime} \neq \emptyset$, then $x \in{\overline{\sum_{k=1}^{m} A_{k}}}^{O \wedge}\left(X^{\prime}\right)$; If $\exists k \in\{1,2, \ldots, m\},[x]_{A_{k}}^{\wedge} \cap X^{\prime}=\emptyset$, then $x \notin{\overline{\sum_{k=1}^{m} A_{k}}}^{O \wedge}\left(X^{\prime}\right)$, and because

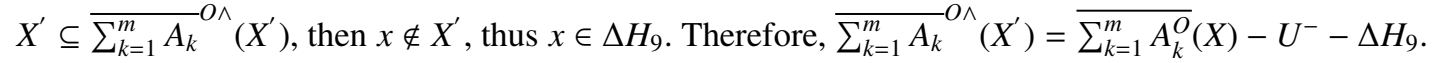

\begin{tabular}{|c|c|c|c|c|}
\hline$U^{\prime}$ & $a_{1}$ & $a_{2}$ & $a_{3}$ & $a_{4}$ \\
\hline$x_{1}$ & 1 & 1 & 1 & 3 \\
\hline$x_{2}$ & 3 & 1 & 1 & 1 \\
\hline$x_{3}$ & 2 & 1 & 1 & 3 \\
\hline$x_{4}$ & 3 & 2 & 1 & 3 \\
\hline$x_{5}$ & 2 & 3 & 2 & 2 \\
\hline$x_{7}$ & 1 & 3 & 2 & 3 \\
\hline
\end{tabular}

Example 4. (Continuation of Example 1) The Table 3 is the reduction of Table 1, let $U^{-}=\left\{x_{6}, x_{8}\right\}, X^{-}=$ $U^{-} \cap X, X^{\prime}=X-X^{-}$. According to Theorem 6 and the results of Example 1, we can calculate the optimistic lower approximation and the upper approximation of $X^{\prime}$ in the new universe as follows:

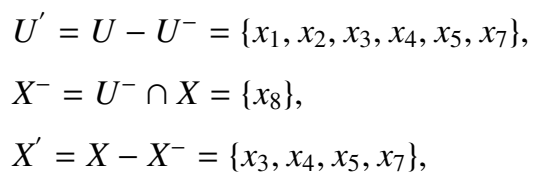

(1) $\sum_{k=1}^{4} A_{k}^{O}(X)=\left\{x_{3}, x_{5}, x_{7}, x_{8}\right\}$;

$\left[x_{3}\right]_{A_{1}} \subseteq X^{\prime}, x_{3} \notin \Delta H_{7} ;$

$\left[x_{5}\right]_{A_{1}} \subseteq X^{\prime}, x_{5} \notin \Delta H_{7}$

$\left[x_{7}\right]_{A_{1}} \nsubseteq X^{\prime},\left[x_{7}\right]_{A_{2}} \nsubseteq X^{\prime},\left[x_{7}\right]_{A_{3}} \nsubseteq X^{\prime},\left[x_{7}\right]_{A_{4}} \nsubseteq X^{\prime}, x_{7} \in \Delta H_{7} ;$

$\left[x_{8}\right]_{A_{1}} \nsubseteq X^{\prime},\left[x_{8}\right]_{A_{2}} \nsubseteq X^{\prime},\left[x_{8}\right]_{A_{3}} \nsubseteq X^{\prime},\left[x_{8}\right]_{A_{4}} \nsubseteq X^{\prime}, x_{8} \in \Delta H_{7} ;$

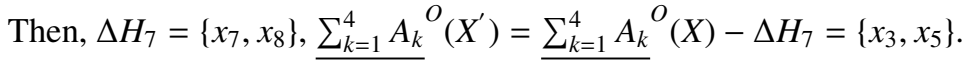

$\left[x_{4}\right]_{A_{1}}^{\wedge} \nsubseteq X^{\prime},\left[x_{4}\right]_{A_{2}}^{\wedge} \subseteq X^{\prime}, x_{4} \in \Delta H_{8} ;$

$\left[x_{7}\right]_{A_{1}}^{\wedge} \nsubseteq X^{\prime},\left[x_{7}\right]_{A_{2}}^{\wedge} \subseteq X^{\prime}, x_{7} \in \Delta H_{8} ;$

Therefore, $\Delta H_{8}=\left\{x_{4}, x_{7}\right\}, \sum_{k=1}^{4} A_{k}{ }^{O \wedge}\left(X^{\prime}\right)=\sum_{k=1}^{4} A_{k}{ }^{O}\left(X^{\prime}\right) \cup \Delta H_{8}=\left\{x_{3}, x_{4}, x_{5}, x_{7}\right\}$.

(2) $\overline{\sum_{k=1}^{4} A_{k}}(X)=\left\{x_{1}, x_{3}, x_{4}, x_{5}, x_{7}, x_{8}\right\}$,

$\left[x_{1}\right]_{A_{1}}^{\wedge} \cap X^{\prime} \neq \emptyset,\left[x_{1}\right]_{A_{2}}^{\wedge} \cap X^{\prime} \neq \emptyset,\left[x_{1}\right]_{A_{3}}^{\wedge} \cap X^{\prime} \neq \emptyset,\left[x_{1}\right]_{A_{4}}^{\wedge} \cap X^{\prime} \neq \emptyset, x_{1} \notin \Delta H_{9} ;$

Therefore, $\Delta H_{9}=\emptyset,{\overline{\sum_{k=1}^{4} A_{k}}}^{O \wedge}\left(X^{\prime}\right)={\overline{\sum_{k=1}^{4} A_{k}}}^{O}(X)-U^{-}-\Delta H_{9}=\left\{x_{1}, x_{3}, x_{4}, x_{5}, x_{7}\right\}$. 
Theorem 7. Give an information system $I S=\langle U, A T, V, f\rangle, A_{1}, A_{2}, \ldots, A_{m} \subseteq A T$, and $\forall X^{\prime} \subseteq U^{\prime}$, the following results hold:

(1) $\sum_{k=1}^{m} A_{k}^{P}\left(X^{\prime}\right) \subseteq \sum_{k=1}^{m} A_{k}^{P \wedge}\left(X^{\prime}\right)$,

(2) ${\overline{\overline{\sum_{k=1}^{m} A_{k}}}}^{P}\left(X^{\prime}\right) \supseteq{\overline{\overline{\sum_{k=1}^{m} A_{k}}}}^{P \wedge}\left(X^{\prime}\right)$.

Proof. (1) $\forall x \in \sum_{k=1}^{m} A_{k}^{P}\left(X^{\prime}\right)$, by Definition 5, we have $\forall k \in\{1,2, \ldots, m\},[x]_{A_{k}} \subseteq X^{\prime}$. When deleting objects, by Definition 9, we have $[x]_{A_{k}}^{\wedge} \subseteq[x]_{A_{k}}$. Thus, $\forall k \in\{1,2, \ldots, m\},[x]_{A_{k}}^{\wedge} \subseteq X^{\prime}$. According to Definition 9, $x \in \sum_{k=1}^{m} A_{k}^{P \wedge}\left(X^{\prime}\right)$. Therefore, $\underline{\sum_{k=1}^{m} A_{k}^{P}}\left(X^{\prime}\right) \subseteq \underline{\sum_{k=1}^{m} A_{k}^{P \wedge}}\left(X^{\prime}\right)$.

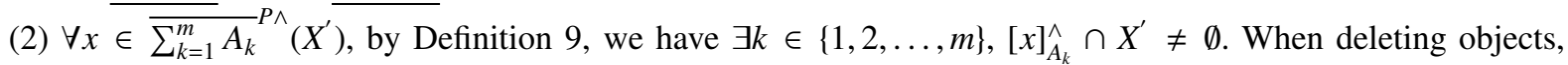
by Definition 9, we have $[x]_{A_{k}}^{\wedge} \subseteq[x]_{A_{k}}$. Thus, $\exists k \in\{1,2, \ldots, m\},[x]_{A_{k}} \cap X^{\prime} \neq \emptyset$. According to Proposition $3, x \in$ ${\overline{\sum_{k=1}^{m} A_{k}}}^{P}\left(X^{\prime}\right)$. Therefore, ${\overline{\sum_{k=1}^{m} A_{k}}}^{P}\left(X^{\prime}\right) \supseteq{\overline{\sum_{k=1}^{m} A_{k}}}^{P \wedge}\left(X^{\prime}\right)$.

Theorem 8. Give an information system $I S=\langle U, A T, V, f\rangle, A_{1}, A_{2}, \ldots, A_{m} \subseteq A T$, let $X^{-}=U^{-} \cap X$, and $\forall X^{\prime} \subseteq U^{\prime}$, $X \subseteq U$, if $X^{\prime}=X-X^{-}$, the following results hold:

(1) $\underline{\sum_{k=1}^{m} A_{k}{ }^{P}}\left(X^{\prime}\right)=\underline{\sum_{k=1}^{m} A_{k}{ }^{P}}(X)-\Delta H_{10}$, $\Delta H_{10}=\left\{x_{i} \in \sum_{k=1}^{m} A_{k}^{P}(X):\left[x_{i}\right]_{A_{k}} \nsubseteq X^{\prime}, \exists k \in\{1,2, \ldots, m\}\right\} ;$

(2) $\underline{\sum_{k=1}^{m} A_{k}^{P \wedge}}{ }^{P}\left(X^{\prime}\right)=\underline{\sum_{k=1}^{m} A_{k}^{P}}\left(X^{\prime}\right) \cup \Delta H_{11}$, $\Delta H_{11}=\left\{x_{i} \in X^{\prime}-\sum_{k=1}^{m} A_{k}^{P}\left(X^{\prime}\right):\left[x_{i}\right]_{A_{k}}^{\wedge} \subseteq X^{\prime}, \forall k \in\{1,2, \ldots, m\}\right\} ;$

(3) ${\overline{\sum_{k=1}^{m} A_{k}}}^{P \wedge}\left(X^{\prime}\right)={\overline{\sum_{k=1}^{m} A_{k}}}^{P}(X)-U^{-}-\Delta H_{12}$, $\Delta H_{12}=\left\{x_{i} \in{\overline{\sum_{k=1}^{m} A_{k}}}^{P}(X)-U^{-}-X^{\prime}:\left[x_{i}\right]_{A_{k}}^{\wedge} \cap X^{\prime}=\emptyset, \forall k \in\{1,2, \ldots, m\}\right\}$.

Proof. (1) $\forall x \in{\underline{\sum_{k=1}^{m} A_{k}}}^{P}(X)$, by Definition 5, we have $\forall k \in\{1,2, \ldots, m\},[x]_{A_{k}} \subseteq X$. If $\forall k \in\{1,2, \ldots, m\}$, $[x]_{A_{k}} \subseteq X^{\prime}$, then $x \in \overline{\sum_{k=1}^{m} A_{k}}{ }^{P}\left(X^{\prime}\right)$; If $\exists k \in\{1,2, \ldots, m\},[x]_{A_{k}} \nsubseteq X^{\prime}$, then $x \in \Delta H_{10}$. Therefore, $\sum_{k=1}^{m} A_{k}^{P}\left(X^{\prime}\right)=$ $\underline{\sum_{k=1}^{m} A_{k}^{P}}(X)-\Delta H_{10}$.

(2) $\forall x \in{\underline{\sum_{k=1}^{m} A_{k}}}^{P \wedge}\left(X^{\prime}\right)$, by Definition 9, we have $\forall k \in\{1,2, \ldots, m\},[x]_{A_{k}}^{\wedge} \subseteq X^{\prime}$. If $\forall k \in\{1,2, \ldots, m\},[x]_{A_{k}} \subseteq X^{\prime}$, then $x \in \underline{\sum_{k=1}^{m} A_{k}{ }^{P}}\left(X^{\prime}\right)$. If $\forall k \in\{1,2, \ldots, m\},[x]_{A_{k}} \nsubseteq X^{\prime}$, because of $\underline{\sum_{k=1}^{m} A_{k}{ }^{P \wedge}}\left(X^{\prime}\right) \subseteq X^{\prime}$, then $x \in \Delta H_{11}$. Therefore,

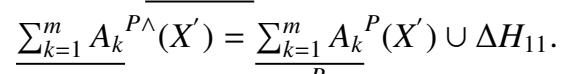

(3) $\forall x \in{\overline{\sum_{k=1}^{m} A_{k}}}^{P}(X)-U^{-}$, by Proposition 3, we have $\exists k \in\{1,2, \ldots, m\},[x]_{A_{k}} \cap X \neq \emptyset$. If $\exists k \in\{1,2, \ldots, m\}$, $[x]_{A_{k}}^{\wedge} \cap X^{\prime} \neq \emptyset$, then $x \in{\overline{\sum_{k=1}^{m} A_{k}}}^{P \wedge}\left(X^{\prime}\right) ;$ If $\forall k \in\{1,2, \ldots, m\},[x]_{A_{k}} \cap X^{\prime}=\emptyset$, then $x \notin{\overline{\sum_{k=1}^{m} A_{k}}}^{P \wedge}\left(X^{\prime}\right)$, and because $X^{\prime} \subseteq{\overline{\sum_{k=1}^{m} A_{k}}}^{P \wedge}\left(X^{\prime}\right)$, then $x \notin X^{\prime}$, thus $x \in \Delta H_{12}$. Therefore, ${\overline{\sum_{k=1}^{m} A_{k}}}^{P \wedge}\left(X^{\prime}\right)={\overline{\sum_{k=1}^{m} A_{k}}}^{P}(X)-U^{-}-\Delta H_{12}$.

Example 5. (Continuation of Example 1) The Table 3 is the reduction of Table 1, let $U^{-}=\left\{x_{6}, x_{8}\right\}, X^{-}=$ $U^{-} \cap X, X^{\prime}=X-X^{-}$. According to Theorem 8 and the results of Example 1, we can calculate the pessimistic lower approximation and the upper approximation of $X^{\prime}$ in the new universe as follows:

$U^{\prime}=U-U^{-}=\left\{x_{1}, x_{2}, x_{3}, x_{4}, x_{5}, x_{7}\right\}$,

$X^{-}=U^{-} \cap X=\left\{x_{8}\right\}$,

$X^{\prime}=X-X^{-}=\left\{x_{3}, x_{4}, x_{5}, x_{7}\right\} ;$

(1) $\sum_{k=1}^{4} A_{k}^{P}(X)=\left\{x_{5}\right\}$,

$\left[x_{5}\right]_{A_{1}} \subseteq X^{\prime},\left[x_{5}\right]_{A_{2}} \nsubseteq X^{\prime}, x_{5} \in \Delta H_{10} ;$ 
Then, $\Delta H_{10}=\left\{x_{5}\right\}, \underline{\sum_{k=1}^{4} A_{k}{ }^{P}}\left(X^{\prime}\right)=\sum_{k=1}^{4} A_{k}{ }^{P}(X)-\Delta H_{10}=\emptyset$.

$\left[x_{3}\right]_{A_{1}}^{\wedge} \subseteq X^{\prime},\left[x_{3}\right]_{A_{2}}^{\wedge} \nsubseteq X^{\prime}, x_{3} \notin \Delta H_{11} ;$

$\left[x_{4}\right]_{A_{1}}^{\wedge} \nsubseteq X^{\prime}, \quad x_{4} \notin \Delta H_{11}$;

$\left[x_{5}\right]_{A_{1}}^{\wedge} \subseteq X^{\prime},\left[x_{5}\right]_{A_{2}}^{\wedge} \subseteq X^{\prime},\left[x_{5}\right]_{\hat{A}_{3}}^{\wedge} \subseteq X^{\prime},\left[x_{5}\right]_{\hat{A}_{4}}^{\wedge} \subseteq X^{\prime}, x_{5} \in \Delta H_{11} ;$

$\left[x_{7}\right]_{A_{1}}^{\wedge} \nsubseteq X^{\prime}, x_{7} \notin \Delta H_{11}$;

Therefore, $\Delta H_{11}=\left\{x_{5}\right\}, \underline{\sum_{k=1}^{4} A_{k}}{ }^{P \wedge}\left(X^{\prime}\right)=\underline{\sum_{k=1}^{4} A_{k}^{P}}\left(X^{\prime}\right) \cup \Delta H_{11}=\left\{x_{5}\right\}$.

(2) ${\overline{\sum_{k=1}^{4} A_{k}}}^{P}(X)=\left\{x_{1}, x_{2}, x_{3}, x_{4}, x_{5}, x_{6}, x_{7}, x_{8}\right\}$,

$\left[x_{1}\right]_{A_{1}}^{\wedge} \cap X^{\prime} \neq \emptyset, x_{1} \notin \Delta H_{12}$;

$\left[x_{2}\right]_{A_{1}}^{\wedge} \cap X^{\prime} \neq \emptyset, x_{2} \notin \Delta H_{12} ;$

Therefore, $\Delta H_{12}=\emptyset,{\overline{\sum_{k=1}^{4} A_{k}}}^{P \wedge}\left(X^{\prime}\right)={\overline{\sum_{k=1}^{4} A_{k}}}^{P}(X)-U^{-}-\Delta H_{12}=\left\{x_{1}, x_{2}, x_{3}, x_{4}, x_{5}, x_{7}\right\}$.

\section{The algorithms for updating multi-granulation approximations while adding or deleting objects}

4.1. The static algorithm for computing multi-granulation approximations

In this subsection, we introduce a static algorithm for multi-granulation rough approximations, which is outlined in Algorithm 1 [20].

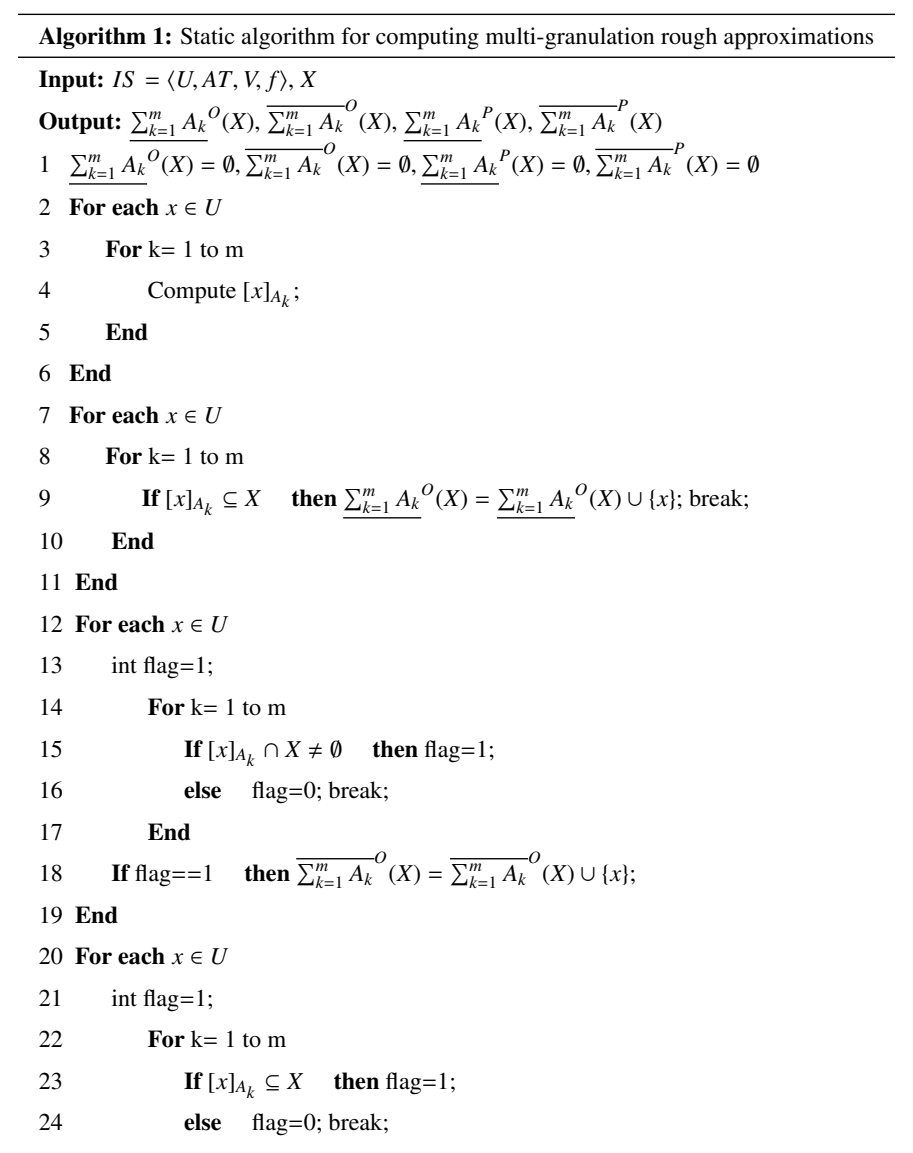




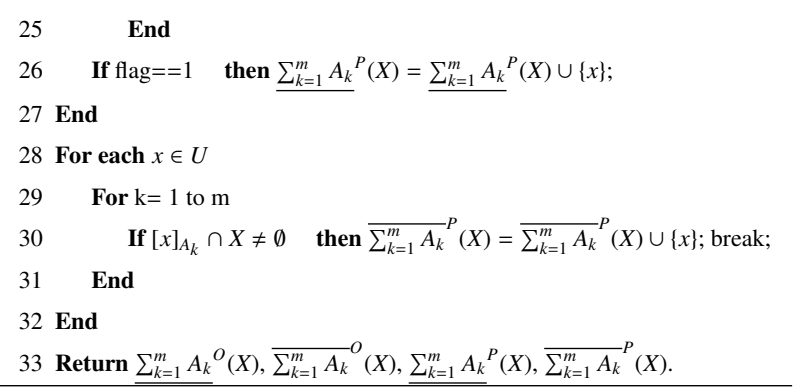

For Algorithm 1, Step 1 initialize the approximations and its time complexity is $O(1)$; Steps 2-6 calculate the equivalence class according to Definition 2, and its time complexity is $O\left(m|U|^{2}\right)$; Steps 7-11 calculate the lower approximation of optimistic multi-granulation rough sets according to Definition 4, and its time complexity is $O(m|U|)$; Steps 12-19 calculate the upper approximation of optimistic multi-granulation rough sets according to Proposition 1, and its time complexity is $O(m|U|)$; Steps 20-27 calculate the lower approximation of pessimistic multi-granulation rough sets according to Definition 5, and its time complexity is $O(m|U|)$; Steps 28-32 calculate the upper approximation of pessimistic multi-granulation rough sets according to Proposition 4, and its time complexity is $O(m|U|)$. Hence, the total time complexity of Algorithm 1 is $O\left(m|U|^{2}\right)$.

4.2 The incremental algorithm for updating multi-granulation approximations while adding objects

In this subsection, we introduce an incremental algorithm for updating multi-granulation rough approximations while adding objects, which is outlined in Algorithm 2.

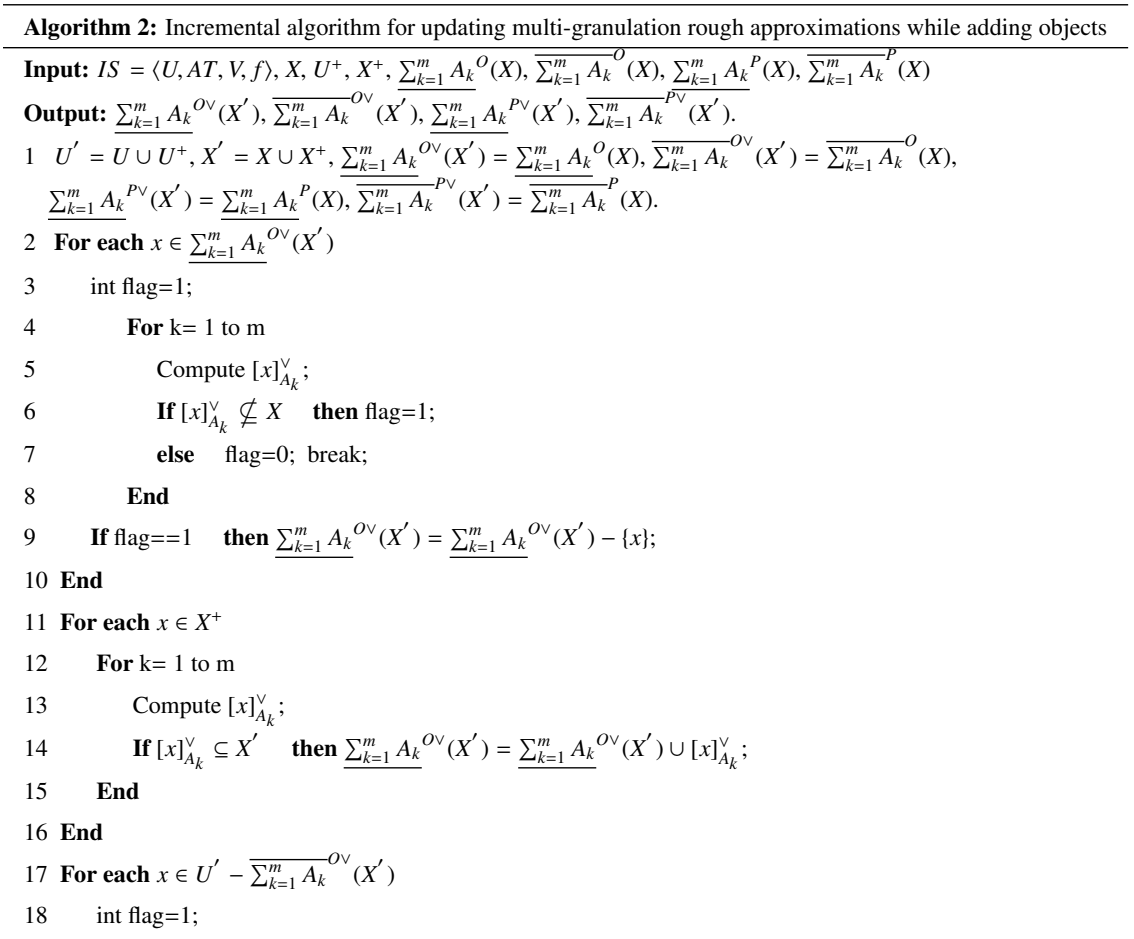




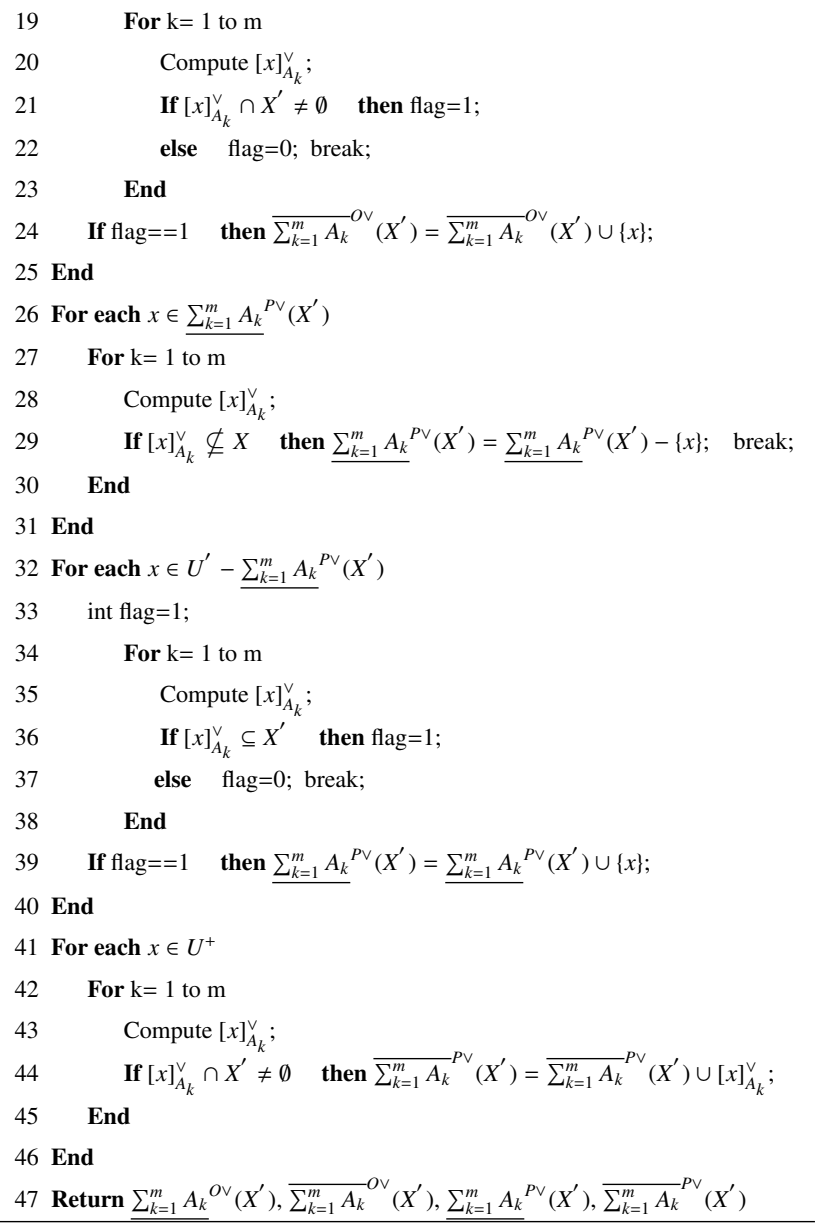

For Algorithm 2, Step 1 calculate the new universe and the new target concept, initialize the approximate sets and its time complexity is $O(1)$; Steps 2-16 calculate the lower approximation of optimistic multi-granulation rough sets according to Definition 6, and its time complexity is $O\left(m\left|U^{\prime}\right|^{2}\right)$; Steps 17-25 calculate the upper approximation of optimistic multi-granulation rough sets according to Definition 6, and its time complexity is $O\left(m\left|U^{\prime}\right|^{2}\right)$; Steps 26-40 calculate the lower approximation of pessimistic multi-granulation rough sets according to Definition 7 , and its time complexity is $O\left(m\left|U^{\prime}\right|^{2}\right)$; Steps 41-46 calculate the upper approximation of pessimistic multi-granulation rough sets according to Definition 7, and its time complexity is $O\left(m\left|U^{\prime}\right|^{2}\right)$. Hence, the total time complexity of Algorithm 2 is $O\left(m\left|U^{\prime}\right|^{2}\right)$, i.e., $O\left(m\left|U \cup U^{+}\right|^{2}\right)$, which is no more than Algorithm 1.

4.3 The incremental algorithm for updating multi-granulation approximations while deleting objects

In this subsection, we introduce an incremental algorithm for updating multi-granulation rough approximations while deleting objects, which is outlined in Algorithm 3.

Algorithm 3: Incremental algorithm for updating multi-granulation rough approximations while deleting objects

Input: $I S=\langle U, A T, V, f\rangle, X, U^{-}, X^{-}, \sum_{k=1}^{m} A_{k}^{O}(X),{\overline{\sum_{k=1}^{m} A_{k}}}^{O}(X), \sum_{k=1}^{m}{A_{k}}^{P}(X),{\overline{\sum_{k=1}^{m} A_{k}}}^{P}(X)$

Output: $\underline{\sum_{k=1}^{m} A_{k}}{ }^{O \wedge}\left(X^{\prime}\right), \overline{\sum_{k=1}^{m} A_{k}}{ }^{O \wedge}\left(X^{\prime}\right), \underline{\sum_{k=1}^{m}} A_{k}^{P \wedge}\left(X^{\prime}\right), \overline{\sum_{k=1}^{m} A_{k}}{ }^{\wedge \wedge}\left(X^{\prime}\right)$. 


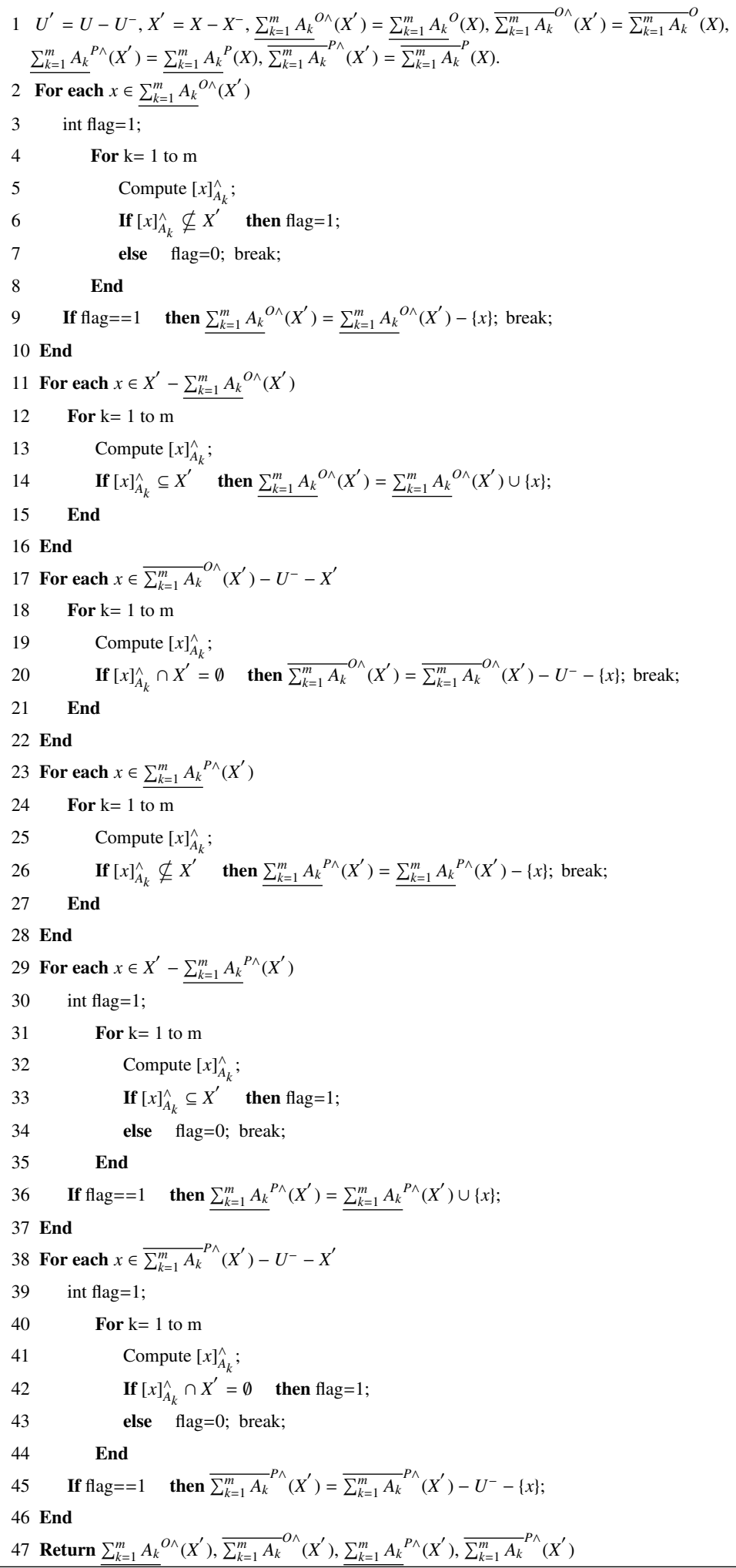

For Algorithm 3, Step 1 calculate the new universe and the new target concept, initialize the approximate sets, 
and its time complexity is $O(1)$; Steps 2-16 calculate the lower approximation of optimistic multi-granulation rough sets according to Definition 8, and its time complexity is $O\left(m\left|U^{\prime}\right|^{2}\right)$; Steps 17-22 calculate the upper approximation of optimistic multi-granulation rough sets according to Definition 8, and its time complexity is $O\left(m\left|U^{\prime}\right|^{2}\right)$; Steps 23-37 calculate the lower approximation of pessimistic multi-granulation rough sets according to Definition 9, and its time complexity is $O\left(m\left|U^{\prime}\right|^{2}\right)$; Steps 38-46 calculate the upper approximation of pessimistic multi-granulation rough sets according to Definition 9, and its time complexity is $O\left(m\left|U^{\prime}\right|^{2}\right)$. Hence, the total time complexity of Algorithm 3 is $O\left(m\left|U^{\prime}\right|^{2}\right)$, i.e., $O\left(m\left|U-U^{-}\right|\right)$, which is no more than Algorithm 1.

\section{Experimental evaluation and analysis}

Many experiments were conducted to evaluate the performance of proposed incremental algorithms. We use 6 date sets available from UCI, the detailed information of these data sets is outlined in Table 4. All the experiments are implemented on a PC with Windows 10, AMD Ryzen5 3550H CPU, $2.10 \mathrm{GHz}$ and $16 \mathrm{~GB}$ memory. The experimental comparisons between Algorithm 1 and Algorithms 2, Algorithm 1 and Algorithms 3 are made in two aspects, respectively. One is to compare the computational time with different sized data sets, the other is to compare the computational time with different updating ratios of the adding or deleting for objects.

Table 4: The description of data sets

\begin{tabular}{cccc}
\hline No. & Data sets & Objects & Attributes \\
\hline 1 & Breast & 286 & 10 \\
2 & Balance & 625 & 5 \\
3 & Solar & 1389 & 13 \\
4 & Chess & 3196 & 37 \\
5 & Mushroom & 8124 & 23 \\
6 & Nursery & 12960 & 8 \\
\hline
\end{tabular}

5.1 Experiments with different sized data sets when adding objects or deleting objects

In this subsection, we compare the computational time of the static and incremental algorithms with the same updating ratio but with different sized data sets when adding objects. We assume that the updating ratio of the incremental or reduced objects is equal to 5\%. Each data set in Table 5 is divided into 10 parts of equal size firstly. Based on the 10 equal sized sub-data sets, and the first part is viewed as the first basic data set, the combination of first part and second part is regarded as the second basic data set, and so on. While adding objects, for each of 10 different basic data sets, we randomly select 5\% of the size of the basic data set from the next subset of data as the new data set to be inserted. While deleting objects, for each of 10 different basic data sets, we randomly select $5 \%$ of the size of the basic data set from the basic data set as the data set to be deleted. 
By comparing the computational time of the static and incremental algorithms, we show the efficiency of proposed incremental algorithms, and the experimental results for computing approximations of the static and incremental algorithms while adding or deleting objects are listed in Tables 5 and 6, respectively. More detailed change trend lines of two proposed algorithms with the increasing size of the data sets are shown in Fig.1 and 2, respectively.

Table 5: The comparison of static and incremental algorithms versus the size of added objects

\begin{tabular}{|c|c|c|c|c|c|c|c|c|c|c|c|c|}
\hline \multirow[b]{2}{*}{ NO. } & \multicolumn{2}{|c|}{ Breast } & \multicolumn{2}{|c|}{ Balance } & \multicolumn{2}{|c|}{ Solar } & \multicolumn{2}{|c|}{ Chess } & \multicolumn{2}{|c|}{ Mushroom } & \multicolumn{2}{|c|}{ Nursery } \\
\hline & Static & Incre. & Static & Incre. & Static & Incre. & Static & Incre. & Static & Incre. & Static & Incre. \\
\hline 1 & 0.112 & 0.039 & 0.233 & 0.042 & 0.713 & 0.121 & 4.534 & 0.494 & 11.540 & 1.143 & 7.715 & 0.850 \\
\hline 2 & 0.252 & 0.045 & 0.396 & 0.067 & 1.366 & 0.169 & 10.707 & 0.635 & 32.352 & 1.741 & 8.765 & 1.321 \\
\hline 3 & 0.338 & 0.059 & 0.493 & 0.069 & 2.194 & 0.215 & 19.587 & 0.929 & 57.341 & 2.079 & 16.513 & 1.425 \\
\hline 4 & 0.405 & 0.055 & 0.641 & 0.096 & 3.350 & 0.243 & 33.600 & 1.075 & 49.075 & 2.372 & 23.339 & 1.737 \\
\hline 5 & 0.498 & 0.058 & 0.780 & 0.104 & 4.443 & 0.257 & 33.904 & 0.927 & 62.718 & 2.329 & 33.817 & 2.142 \\
\hline 6 & 0.513 & 0.053 & 0.964 & 0.115 & 5.822 & 0.293 & 35.181 & 1.239 & 88.771 & 2.651 & 46.082 & 2.657 \\
\hline 7 & 0.625 & 0.082 & 1.104 & 0.135 & 7.082 & 0.367 & 43.418 & 1.784 & 117.439 & 3.289 & 59.327 & 3.332 \\
\hline 8 & 0.720 & 0.073 & 1.298 & 0.142 & 8.237 & 0.389 & 47.542 & 1.590 & 149.876 & 3.575 & 74.426 & 3.901 \\
\hline 9 & 0.851 & 0.065 & 1.518 & 0.146 & 11.262 & 0.491 & 51.219 & 1.967 & 184.151 & 4.156 & 90.745 & 4.437 \\
\hline 10 & 0.903 & 0.076 & 1.710 & 0.164 & 13.533 & 0.574 & 59.941 & 1.995 & 218.581 & 4.560 & 110.957 & 4.982 \\
\hline
\end{tabular}
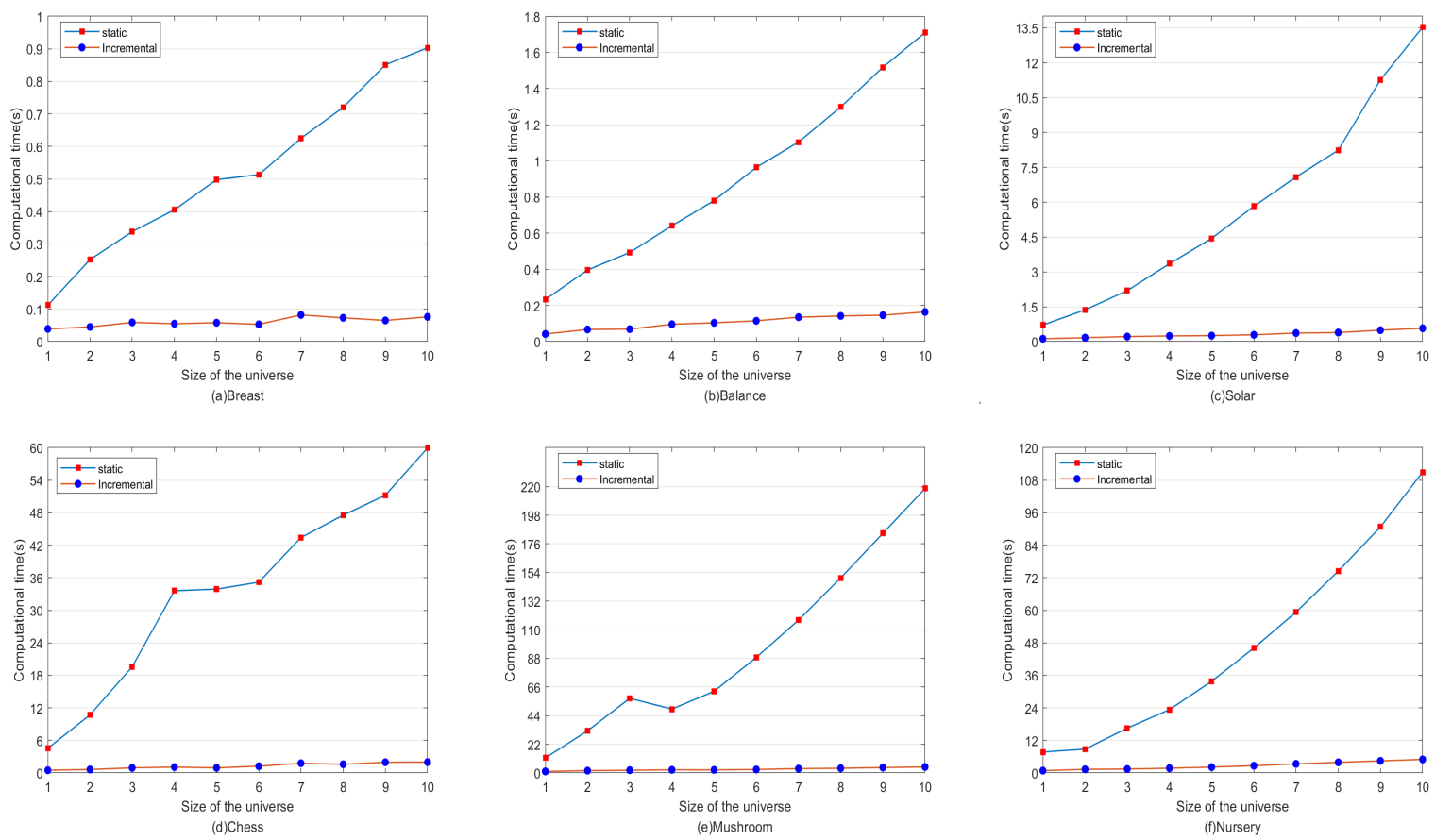

Figure 1: Computational time of static and incremental algorithms versus the size of added objects.

In each sub-figure of Fig.1 and 2, the x-coordinate is the size of the data set, the y-coordinate is the computational time, in addition, square and point lines denote the computational time of static and incremental algorithms, 
Table 6: The comparison of static and incremental algorithms versus the size of deleted objects

\begin{tabular}{|c|c|c|c|c|c|c|c|c|c|c|c|c|}
\hline \multirow[b]{2}{*}{ NO. } & \multicolumn{2}{|c|}{ Breast } & \multicolumn{2}{|c|}{ Balance } & \multicolumn{2}{|c|}{ Solar } & \multicolumn{2}{|c|}{ Chess } & \multicolumn{2}{|c|}{ Mushroom } & \multicolumn{2}{|c|}{ Nursery } \\
\hline & Static & Incre. & Static & Incre. & Static & Incre. & Static & Incre. & Static & Incre. & Static & Incre. \\
\hline 1 & 0.085 & 0.045 & 0.231 & 0.134 & 0.693 & 0.347 & 4.148 & 1.916 & 10.154 & 4.668 & 6.662 & 2.973 \\
\hline 2 & 0.187 & 0.084 & 0.360 & 0.205 & 1.288 & 0.555 & 10.132 & 4.754 & 28.341 & 13.830 & 16.737 & 7.876 \\
\hline 3 & 0.279 & 0.103 & 0.463 & 0.246 & 2.009 & 0.860 & 17.732 & 8.403 & 51.744 & 24.618 & 16.039 & 7.747 \\
\hline 4 & 0.337 & 0.147 & 0.602 & 0.340 & 3.189 & 1.319 & 29.870 & 14.690 & 61.151 & 34.013 & 24.972 & 11.692 \\
\hline 5 & 0.364 & 0.182 & 0.769 & 0.414 & 4.006 & 1.880 & 37.275 & 17.269 & 62.044 & 30.238 & 34.973 & 16.064 \\
\hline 6 & 0.483 & 0.195 & 0.873 & 0.426 & 5.446 & 2.427 & 32.431 & 11.376 & 89.346 & 44.344 & 45.171 & 21.099 \\
\hline 7 & 0.618 & 0.240 & 1.121 & 0.497 & 6.436 & 3.034 & 36.615 & 15.061 & 107.902 & 59.253 & 61.046 & 27.474 \\
\hline 8 & 0.683 & 0.261 & 1.297 & 0.559 & 7.985 & 3.699 & 46.094 & 19.956 & 147.401 & 70.713 & 72.578 & 33.901 \\
\hline 9 & 0.760 & 0.319 & 1.506 & 0.695 & 10.373 & 4.614 & 45.153 & 24.915 & 167.690 & 91.833 & 85.091 & 39.344 \\
\hline 10 & 0.855 & 0.369 & 1.688 & 0.685 & 12.108 & 5.258 & 55.242 & 26.417 & 253.503 & 109.896 & 103.568 & 48.805 \\
\hline
\end{tabular}
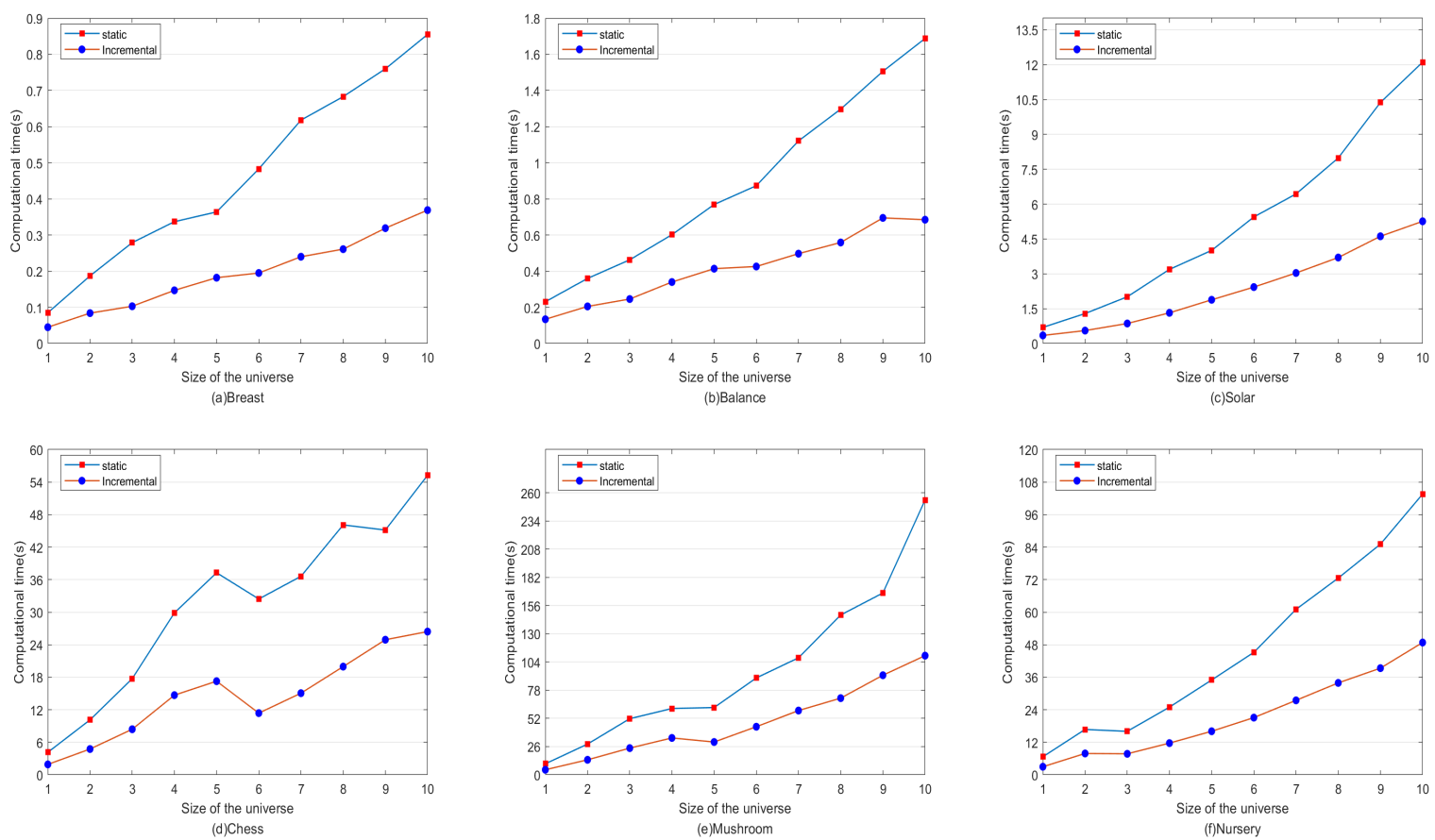

Figure 2: Computational time of static and incremental algorithms versus the size of deleted objects.

respectively. From Fig.1, it is easy to see the computation time of Algorithm 1 and Algorithm 2 usually increases with the increase of the size of data sets, while the proposed incremental algorithm is consistently faster than the static algorithm, and the differences of efficiency are profoundly larger when the size of the data sets increases. From Fig.2, it is easy to see the computation time of Algorithm 1 and Algorithm 3 usually increases with the increase of the size of data sets, while the proposed incremental algorithm is consistently faster than the static algorithm.

5.2 Experiments with different update rations of data sets when adding or deleting objects 
In this subsection, we compare the computational time of the static and incremental algorithms with the same size of the basic data sets but with different updating ratios when adding or deleting objects. For the situation of adding objects, We select half of the universe randomly as the basic data sets, and then select $R_{a}^{i}$ of the objects from the remaining $50 \%$ objects of the universe as the added objects, $R_{a}^{i}$ is equal to $10 \%, 20 \%, \ldots, 100 \%$, respectively. For the situation of deleting objects, we select whole universe as the basic data sets, and then select $R_{a}^{i}$ of the objects from the basic data sets as the deleted objects, $R_{a}^{i}$ is equal to $10 \%, 20 \%, \ldots$., $90 \%$, respectively. Algorithms 2 and 3 are applied to update the approximations, respectively.

By comparing the computational time of the static and incremental algorithms, we show the efficiency of proposed incremental algorithms, and the experimental results for computing approximations of the static and incremental algorithms while adding or deleting objects are listed in Tables 7 and 8, respectively. More detailed change trend lines of two proposed algorithms with the increasing size of the data sets are shown in Fig. 3 and 4, respectively.

Table 7: The comparison of static and incremental algorithms versus the updating rations of added objects

\begin{tabular}{|c|c|c|c|c|c|c|c|c|c|c|c|c|}
\hline \multirow[b]{2}{*}{ NO. } & \multicolumn{2}{|c|}{ Breast } & \multicolumn{2}{|c|}{ Balance } & \multicolumn{2}{|c|}{ Solar } & \multicolumn{2}{|c|}{ Chess } & \multicolumn{2}{|c|}{ Mushroom } & \multicolumn{2}{|c|}{ Nursery } \\
\hline & Static & Incre. & Static & Incre. & Static & Incre. & Static & Incre. & Static & Incre. & Static & Incre. \\
\hline 1 & 0.412 & 0.117 & 0.870 & 0.193 & 4.896 & 0.962 & 36.793 & 7.029 & 98.507 & 17.244 & 42.701 & 8.057 \\
\hline 2 & 0.501 & 0.192 & 1.070 & 0.326 & 5.490 & 1.890 & 35.280 & 9.570 & 112.667 & 33.899 & 59.677 & 16.957 \\
\hline 3 & 0.531 & 0.254 & 1.118 & 0.422 & 6.155 & 2.753 & 39.778 & 16.161 & 125.729 & 53.827 & 60.791 & 22.318 \\
\hline 4 & 0.639 & 0.321 & 1.199 & 0.555 & 6.856 & 3.804 & 41.056 & 27.417 & 138.285 & 70.254 & 70.967 & 32.738 \\
\hline 5 & 0.671 & 0.358 & 1.307 & 0.756 & 7.584 & 4.810 & 40.320 & 25.279 & 151.907 & 83.778 & 82.254 & 40.761 \\
\hline 6 & 0.754 & 0.467 & 1.271 & 0.749 & 8.469 & 6.014 & 42.591 & 30.225 & 168.730 & 95.654 & 88.581 & 48.797 \\
\hline 7 & 0.835 & 0.569 & 1.442 & 0.913 & 9.247 & 6.946 & 54.640 & 41.294 & 168.863 & 113.764 & 93.026 & 64.467 \\
\hline 8 & 0.843 & 0.650 & 1.479 & 1.028 & 11.863 & 9.504 & 59.906 & 49.553 & 182.185 & 133.639 & 99.211 & 72.585 \\
\hline 9 & 0.883 & 0.726 & 1.567 & 1.143 & 12.513 & 10.347 & 67.339 & 57.147 & 202.941 & 172.190 & 112.888 & 85.562 \\
\hline 10 & 0.914 & 0.996 & 1.680 & 1.303 & 14.948 & 11.730 & 80.699 & 71.612 & 218.138 & 186.150 & 126.808 & 107.642 \\
\hline
\end{tabular}

Table 8: The comparison of static and incremental algorithms versus the updating rations of deleted objects

\begin{tabular}{|c|c|c|c|c|c|c|c|c|c|c|c|c|}
\hline \multirow[b]{2}{*}{ NO. } & \multicolumn{2}{|c|}{ Breast } & \multicolumn{2}{|c|}{ Balance } & \multicolumn{2}{|c|}{ Solar } & \multicolumn{2}{|c|}{ Chess } & \multicolumn{2}{|c|}{ Mushroom } & \multicolumn{2}{|c|}{ Nursery } \\
\hline & Static & Incre. & Static & Incre. & Static & Incre. & Static & Incre. & Static & Incre. & Static & Incre. \\
\hline 1 & 0.824 & 0.413 & 1.640 & 0.711 & 11.195 & 5.275 & 63.688 & 31.734 & 200.307 & 88.628 & 98.763 & 45.732 \\
\hline 2 & 0.734 & 0.387 & 1.364 & 0.576 & 8.627 & 3.794 & 50.901 & 24.277 & 154.009 & 80.044 & 97.667 & 39.605 \\
\hline 3 & 0.727 & 0.334 & 1.122 & 0.457 & 7.185 & 3.218 & 44.443 & 20.893 & 133.876 & 64.992 & 75.115 & 34.136 \\
\hline 4 & 0.550 & 0.323 & 1.005 & 0.435 & 5.545 & 2.680 & 30.674 & 17.629 & 103.105 & 51.651 & 62.277 & 29.929 \\
\hline 5 & 0.479 & 0.299 & 0.731 & 0.348 & 4.534 & 2.031 & 35.032 & 14.685 & 72.416 & 36.850 & 44.945 & 22.278 \\
\hline 6 & 0.407 & 0.259 & 0.589 & 0.273 & 3.312 & 1.426 & 33.109 & 14.601 & 52.367 & 25.079 & 30.884 & 15.973 \\
\hline 7 & 0.315 & 0.167 & 0.486 & 0.240 & 2.308 & 1.027 & 22.072 & 9.678 & 44.052 & 28.147 & 21.062 & 10.301 \\
\hline 8 & 0.256 & 0.143 & 0.376 & 0.133 & 1.447 & 0.649 & 12.432 & 5.529 & 26.338 & 16.277 & 16.507 & 9.002 \\
\hline 9 & 0.113 & 0.089 & 0.202 & 0.086 & 0.657 & 0.300 & 5.758 & 1.907 & 12.638 & 5.132 & 8.544 & 3.274 \\
\hline
\end{tabular}



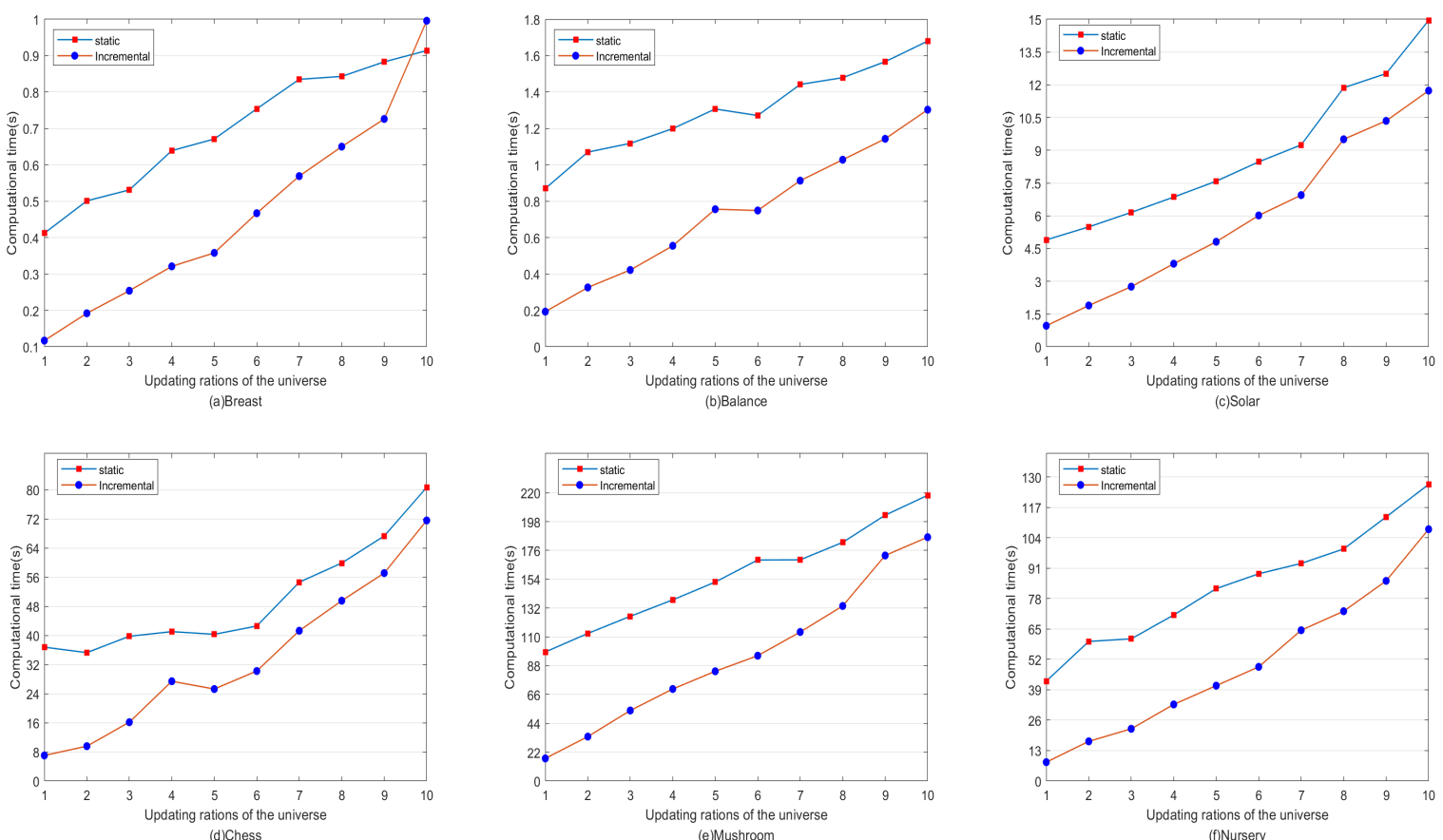

Figure 3: Computational time of static and incremental algorithms versus the updating rations of added objects.
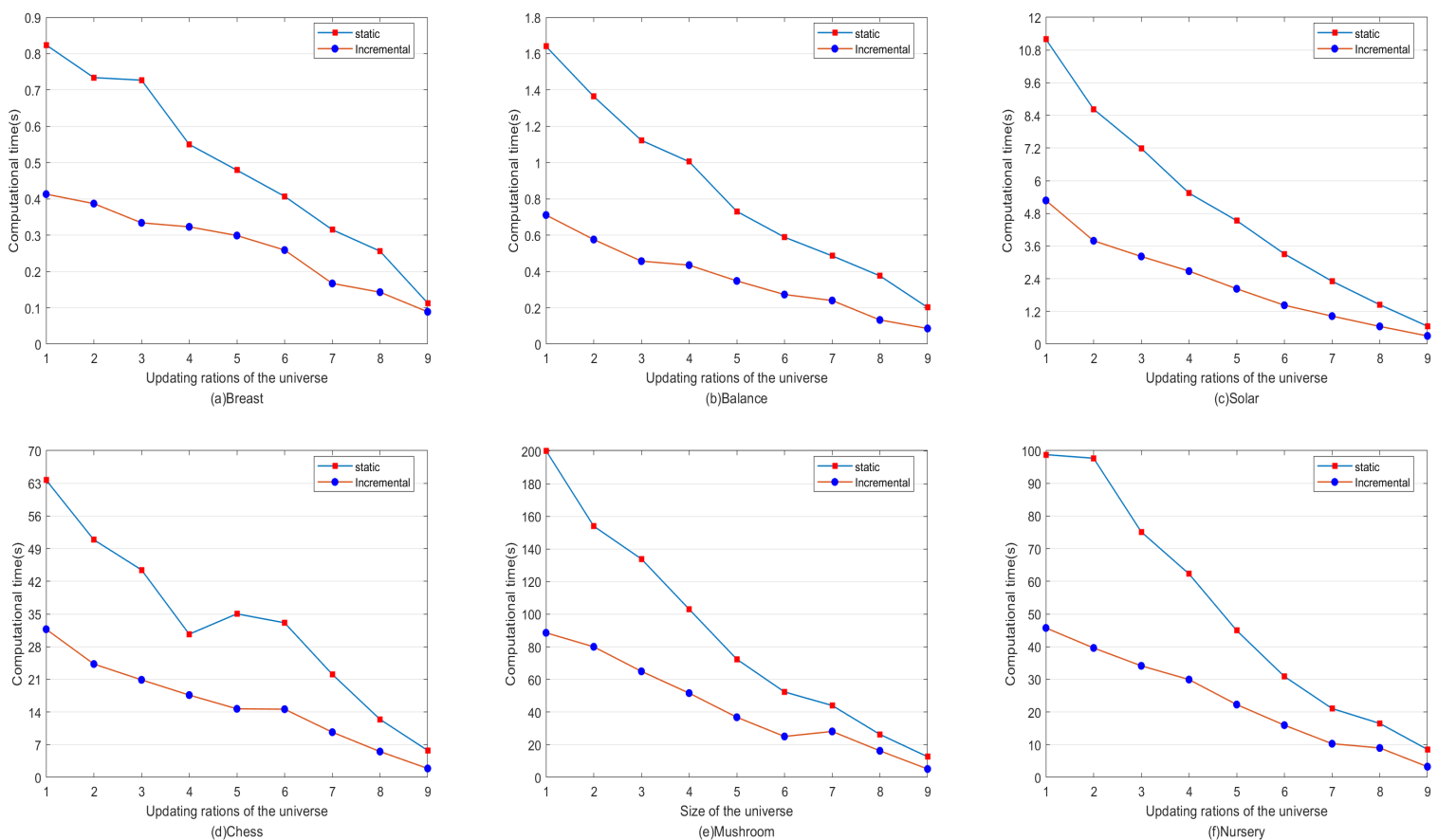

Figure 4: Computational time of static and incremental algorithms versus the updating rations of deleted objects.

In each sub-figure of Fig. 3 and 4, the x-coordinate is the updating ratios of the data set, the y-coordinate is the computational time, in addition, square and point lines denote the computational time of static and incremental al- 
gorithms, respectively. From Fig.3, it is easy to see the computation time of Algorithm 1 and Algorithm 2 usually increases with the increase of the updating ratios of the data sets, while the proposed incremental algorithm is consistently faster than the static algorithm, and the differences of efficiency are profoundly larger when the updating ratios of the data sets decreases. From Fig.4, it is easy to see the computation time of Algorithm 1 and Algorithm 3 usually decreases with the increase of the updating ratios of data sets, while the proposed incremental algorithm is consistently faster than the static algorithm.

\section{Conclusions}

In this paper, incremental approximations are presented. With adding or deleting of objects, the approximations can be calculated incrementally without computing fully updated data set, they are used to design incremental algorithms for updating approximations. Our theoretical analysis guarantees the results of incremental algorithms are right, experimental studies using 6 UCI data sets shows that proposed incremental algorithms can significantly reduce computing time for calculating approximations with dynamic data. Further investigations are planned, such as tolerance-based multi-granulation incremental approximations, or multi-granulation incremental approximations with attributes and objects changed at the same time.

Acknowledgments This work is supported by the Nature Science Foundation of Shanxi Province(No. 201901D111280).

\section{Compliance with ethical standards}

Conflict of interest Author Hong Wang declares that he has no conflict of interest. Author Jingtao Guan declares that she has no conflict of interest.

Ethical approval This article does not contain any studies with human participants or animals performed by any of the authors.

\section{References}

[1] Z.Pawlak, Rough sets, International Journal of Computer and Information Sciences, 11(5)(1982) 341-356.

[2] V.S.Ananthanarayana, M.Narasimha Murty, D.K.Subramanian, Tree structure for efficient data mining using rough sets, Pattern Recognition Letters, 24(2003) 851-862.

[3] J.F.Peters, A.Skowron, A rough set approach to knowledge discovery, International Journal of Intelligent Systems, 17(2)(2002) 109-112.

[4] J.Stepaniuk, K.Kierzkowska, Hybrid classifier based on rough sets and neural networks, Electronic Notes in Theoretical Computer Science, $82(2003) 228-238$.

[5] J.Stepaniu, Relational data and rough sets, Fundamenta Informaticae, 79(2007) 525-539.

[6] A.Skowron, J.Stepaniuk, R.Swiniarski, Approximation spaces in roughgranular computing, Fundamenta Informaticae, $100(2010) 141-157$.

[7] W.H.Shu, W.B.Qian, Y.H.Xie, Incremental approaches for feature selection from dynamic data with the variation of multiple objects, Knowledge-Based Systems, 163(2019) 320-331.

[8] Y.Fan, T.Tseng, C.Chem, C.Huang, Rule induction based on an incremental rough set, Expert Systems with Applications, 36(9)(2009) 1143911450 .

[9] J.Zhang, T.R.Li, D.Ruan, D.Liu, Neighborhood rough sets for dynamic data mining, International Joumnal of Intelligence Systems, 27(2012) 317-342. 
[10] H.M.Chen, T.R.Li, D.Ruan, J.H.Lin, C.X.Hu, A rough-set based incremental approach for updating approximations under dynamic maintenance environments, IEEE Trans-actions on Knowledge and Data Engineering, 25(2)(2013) 274-284.

[11] S.Y.Li, T.R.Li, D.Liu, Dynamic maintenance of approximations in dominance-basedrough set approach under the variation of the object set, International Journal of Intelligent Systems, 28(2013) 729-751.

[12] J.Hu, T.R.Li, H.M.Chen, A.P.Zeng, An incremental learning approach for updating approximations in rough set model over dual universes, International Journal of Intelligent Systems, 30(8)(2015) 923-947.

[13] C.Luo, T.R.Li, H.M.Chen, H.Fujita, Z.Yi, Efficient updating of probabilistic approximations with incremental objects, Knowledge-Based Systems, 109(2016) 71-83.

[14] C.X.Hu, L.Zhang, Efficient approaches for maintaining dominance-based multigranulation approximations with incremental granular structures, International Journal of Approximate Reasoning, 126(2020) 202-227.

[15] G.M.Lang, D.Q.Miao, M.J.Cai, Z.F.Zhang, Incremental approaches for updating reducts in dynamic covering information systems, Knowledge-Based Systems, 134(2017) 85-104.

[16] C.X.Hu, S.X.Liu, G.X.Liu, Matrix-based approaches for dynamic updating approximations in multigranulation rough sets, Knowledge-Based Systems, 122(2017) 51-63.

[17] Y.Cheng, The incremental method for fast computing the rough fuzzy approximations, Data \& Knowledge Engineering, 70(1)(2011) 84-100.

[18] J.B.Zhang, T.R.Li, D.Ruan, D.Liu, Rough sets based matrix approaches with dynamic attribute variation in set-valued information systems, International Journal of Approximate Reasoning, 53(4)(2012) 620-635.

[19] S.Y.Li, T.R.Li, D.Liu, Incremental updating approximations in dominance-based rough sets approach under the variation of the attirbute set, Knowledge-Based Systems, 40(2013) 17-26.

[20] X.B.Yang, Y.Qi, H.L.Yu, X.N.Song, J.Y.Yang, Updating multigranulation rough approximations with increasing of granular structures, Knowledge-Based Systems, 64(2014) 59-69.

[21] H.M.Chen, T.R.Li, S.J.Qiao, A rough set based dynamic maintenance approach for approximations in coarsening and refining attribute values, International Journal of Intelligent Systems, 25(2010) 1005-1026

[22] H.M.Chen, T.R.Li, D.Ruan, Maintenance of approximations in incomplete ordered decision systems while attribute values coarsening or refining, Knowledge-Based Systems, 31(7)(2012) 140-161.

[23] F.Wang, J.Y.Liang, C.Y.Dang, Attribute reduction for dynamic data sets, Applied Soft Computing, 13(1)(2013) 676-689.

[24] H.M.Chen, T.R.Li, C.Luo, S.J.Horng, G.Y.Wang, A rough set-based method for updating decision rules on attribute values coarsening and refining, IEEE Transactions on Knowledge and Data Engineering, 26(12)(2014) 2886-2899.

[25] S.Y.Li, T.R.Li, Incremental update of approximations in dominance-based rough sets approach under the variation of attribute values, Information Sciences, 294(2015) 348-361.

[26] C.Luo, T.R.Li, H.M.Chen, L.X.Lu, Fast algorithms for computing rough approximations in set-valued decision systems while updating criteria values, Information Sciences, 299(2016) 221-242.

[27] A.P.Zeng, T.R.Li, J.Hu, H.M.Chen, C.Luo, Dynamical updating fuzzy rough approximations for hybrid data under the variation of attribute values, Information Sciences, 378(2017) 363-388.

[28] C.X.Hu, S.X.Liu, X.L.Huang, Dynamic updating approximations in multigranulation rough sets while refining or coarsening attribute values, Knowledge-Based Systems, 130(2017) 62-73.

[29] Y.H.Qian, J.Y.Liang, Y.Y.Yao, C.Y.Dang, MGRS: A multi-granulation rough set, Information Sciences, 180(2010) 949-970.

[30] Y.H.Qian, J.Y.Liang, C.Y.Dang, Incomplete multigranulation rough set, IEEE Transactions on Systems Man and Cybernetics Part A, 40(2)(2010) 420-431.

[31] G.P.Lin, Y.H.Qian, J.J.Li, NMGRS:Neighborhood-based multigranulation rough sets, International Journal of Approximate Reasoning, 53(7)(2012)1080-1093.

[32] Y.H.Qian, H.Zhang, Y.L.Sang, J.Y.Liang, Multigranulation decision-theoretic rough sets, International Journal of Approximate Reasoning, 55(1)(2014) 225-237. 
[33] B.Huang, C.X.Guo, Y.L.Zhuang, H.X.Li, X.Z.Zhou, Intuitionistic fuzzy multigranulation rough sets, Information Sciences, 277(2014) 299320 .

[34] T.Feng, J.S.Mi, Variable precision multigranulation decision-theoretic fuzzy rough sets, Knowledge-Based Systems, 91(2016) 93-101.

[35] Y.H.Qian, X.Y.Liang, G.P.Lin, Q.Guo, J.Y.Liang, Local multigranulation decision-theoretic rough sets, International Journal of Approximate Reasoning, 82(2017) 119-137.

[36] W.H.Xu, W.T.Wang, X.T.Zhang, Generalized multigranulation rough sets and optimal granularity selection, Granular Computing, 2(2017) $271-288$. 